TRANSACTIONS OF THE

AMERICAN MATHEMATICAL SOCIETY

Volume 361, Number 3, March 2009, Pages 1241-1268

S 0002-9947(08)04764-8

Article electronically published on October 10, 2008

\title{
THE HELE-SHAW PROBLEM AS A "MESA" LIMIT OF STEFAN PROBLEMS: EXISTENCE, UNIQUENESS, AND REGULARITY OF THE FREE BOUNDARY
}

\author{
IVAN A. BLANK, MARIANNE K. KORTEN, AND CHARLES N. MOORE
}

\begin{abstract}
We study a Hele-Shaw problem with a mushy region obtained as a mesa type limit of one-phase Stefan problems in exterior domains. We deal with both Neumann and Dirichlet data and show pointwise convergence of the Stefan solutions to the Hele-Shaw solution. We make no assumptions on the geometry, topology, or connectivity of the injection slot.
\end{abstract}

\section{INTRODUCTION}

Given a bounded domain $D \in \mathbb{R}^{n}$ with smooth boundary $\partial D$, a finite set of closed curves $\left\{s_{0}^{j}(x)=0\right\}$ such that $D$ is contained in the union of their interiors, and a continuous function $p(x, t)$ defined on $\partial D \times(0, \infty)$, the classical Hele-Shaw problem with Dirichlet data is frequently formulated as follows: Find a function $V(x, t)$ and a family of domains $S(t)$ (each of which contain $D$ ) with $\partial S(t)=$ $\{x: s(x, t)=0\}=\{x: t=\tilde{s}(x)\}$ and such that

$$
\begin{array}{ll}
\Delta_{x} V=0 & (x, t) \in S(t) \backslash D \\
V(x, t)=0 & (x, t) \in \partial S(t) \\
V(x, t)=p(x, t) & (x, t) \in \partial D \times(0, \infty) \\
\nabla_{x} V \cdot \nabla_{x} s=\frac{\partial s}{\partial t} & (x, t) \in \partial S(t) \\
\partial S(0)=\bigcup_{j}\left\{s_{0}^{J}(x)=0\right\} . &
\end{array}
$$

The Hele-Shaw problem is occasionally referred to in the literature as the quasistatic or quasi-stationary Stefan problem, although in those cases the partial differential equation often includes lower order terms and the free boundary condition often includes a curvature term.

This problem models the advance of the slick formed by injecting oil between two nearby plates, and has further been used in injection molding (used in turn in the packaging industry, and more generally for the production of plastic components, for example interior pieces of cars and aircraft), in electrochemical machining (see

Received by the editors October 18, 2006.

2000 Mathematics Subject Classification. Primary 76D27, 35K65, 49J40.

Key words and phrases. Mesa problem, Hele-Shaw problem, Stefan problem, free boundary, mushy region, singular limit.

(C)2008 American Mathematical Society Reverts to public domain 28 years from publication 
MR] ), and even to predict tumor growth (see $[\mathrm{BF}]$ ). Sometimes the normal derivative of $V$ at the "slot" $\partial D$ is prescribed, or curvature dependent terms are included in the free boundary condition. Among the important questions concerning the Hele-Shaw problem are finding a suitable weak formulation, studying the regularity of $V(x, t)$ in $t$, and determining the regularity of the free boundary $t=s(x)$. Another question which has long attracted interest is whether a Hele-Shaw problem could have a "mushy" region.

Because of changes in the topology of the advancing slick, the need for a suitable definition of weak solution is one of the most important concerns in the study of Hele-Shaw models. In 1981 Elliott and Janovský introduced a notion of weak solution to the Hele-Shaw problem and showed that there exists a unique solution in their setup. (See [EJ].) To arrive at their notion of weak solution, one would take the integral in time of a classical solution to Hele-Shaw (i.e. take the so-called Baiocchi transform of the function $V$ in Equation (1.1)), and then look at the equations satisfied after "testing" the Baiocchi transform against a suitable class of functions. It turns out that after the integration in time, the new functions are solutions of obstacle problems at each fixed time, and therefore there is a large theory which can be applied. This fact was presumably a major factor in making this notion of weak solution not only the most popular, but as far as we can tell, the only notion of weak solution used in the 1980s and 1990s. (See DF for a similar idea for the ill-posed version of the problem.)

Now the bad news about this notion of a solution is that there is no a priori reason why the derivative in time of this weak solution is even a function. In the approach used in this paper, the weak solutions satisfy equations that would come from immediately testing classical Hele-Shaw solutions against suitable functions. Only after we have existence and uniqueness of our weak solutions do we integrate in time and appeal to obstacle problem theory to get regularity of the free boundary. We also automatically get weak solutions of the type studied by Elliott and Janovský when we integrate our weak solutions in time. The notion of weak solution which we employ is seen only very recently in work by Gil and Quirós, and Gil, Quirós, and Vázquez (see GQV and the references therein), although they appear to need constant boundary data in order to make the theory work. We will compare our work with theirs in a more detailed manner after we first introduce the notion of a "Mesa" problem.

The Mesa problem describes the limit pattern $\lim _{m \rightarrow \infty} u_{m}$ of solutions $u_{m}$ of, say, the porous medium equation, when the initial data are held fixed. This problem first appeared in connection with the modeling of problems related to transistors (see [EHKO]). Caffarelli and Friedman studied the initial value problem in $\mathbb{R}^{n} \times[0, T]$ in $[\mathrm{CF}$. They proved that the limit exists, that it is independent of the chosen subsequence, that it is independent of time, that it is equal to the characteristic function of one set plus the initial data times the characteristic function of the complement of that set, and finally that that set can be characterized as the noncoincidence set of a variational inequality. Further developments showed that the same conclusions hold for the limit when $u^{m}$ is replaced by a fairly general sequence of monotone constitutive functions $\phi_{m}(u)$ with the same limiting graph and with $\phi_{m}(0)=0$ (see Friedman and Huang's paper [FH] for example); moreover, this behavior is a property of fairly general semigroups (see [I], [BEG]). A Mesa problem for a Stefan type equation which gives rise to a mushy region was 
studied in BoKM]. For the Mesa problem we study in this paper we show all of the properties shown by $[\mathrm{CF}$ mentioned above, except that our limits will not be independent of time. This evolution in time is natural since we work in an outer domain where the inner boundary data serves as a source. We also note that both Caffarelli and Friedman, and Friedman and Huang, needed assumptions about the geometry of the level sets of $u_{I}$ in order to conclude further regularity of their free boundaries, while we do not.

In this paper we exploit a mesa limit setting in an outer domain $D^{c}$ to obtain naturally a weak formulation of the Hele-Shaw problem (with Dirichlet condition as in Equation (1.1) on the slot as above). The use of one-phase Stefan problems with "mushy" regions and with increasing diffusivities naturally produces a mushy region when we permit initial data $u_{I}$ for the approximating problems to take values in the interval $[0,1]$. These $u_{I}$ can be thought of as generalized characteristic functions. Another aspect of our approach which is extremely attractive is the fact that changes in the topology of the "wet" region do not interfere with the construction. In short, whereas other authors have made a priori assumptions which ensure that their free boundary stays smooth, we have been able to show existence of weak solutions for all time, regardless of the possible changes in topology. (Note that in $[\mathrm{CF}]$ and $[\mathrm{FH}]$ the authors assume that the level sets of their data are starlike with respect to the origin, and in DL log concavity of initial data is assumed to guarantee existence and smoothness of the solutions.) Indeed there are some very natural problems arising in the applications where the topology should change. Consider for example the problem of what happens with Hele-Shaw flow around an obstacle. In this case, there is automatically a change in topology when the flow meets itself on the other side. An interesting question is whether or not an air bubble will be left behind in the wake of the obstacle. Another obvious problem from applications where the topology will change is if there are multiple injection slots. In fact, this paper already deals with the second situation, since we never assume that $D$ is connected.

Recently, Gil, Quirós, and Vázquez have studied the Mesa limit of solutions of the porous medium equation on exterior domains and were able to get convergence without the geometric assumptions that Caffarelli, Friedman, and Huang had imposed. (See $[\mathrm{GQV}]$ and the references therein.) As indicated before, their work also employs a notion of weak solution of Hele-Shaw which does not a priori involve an integration in time. The present work runs somewhat parallel to some parts of these works, but there are several advantages of our methods. First, we have more general boundary data, and in particular the dependence in time of $p$ seems to make things much more complicated. Indeed, the energy estimates become more involved and some very useful monotonicity is lost when $p$ is not assumed to be nondecreasing in time. To get a measure of the added difficulty, one can simply compare the length and depth of the fourth and fifth sections of the present work. In the fourth section we assume that $p$ is nondecreasing in time and very quickly get pointwise monotone convergence, whereas in the fifth section where we do not assume any monotonicity in time of the data, we need a much more circuitous route to establish pointwise convergence almost everywhere. In order to deal with Neumann data, however, this generalization is absolutely necessary. Since the Hele-Shaw problem is more often formulated with Neumann data when it arises in applications, it is unsatisfactory to create a mathematical theory for the Dirichlet data which does 
not extend to the Neumann case. In the Neumann case there is no reason to expect that boundary Dirichlet data will be increasing in time. Second, we have a slightly weaker notion of solution and stronger convergence than the solutions and convergences of Gil and Quirós. Our convergence is in all $L^{p}$ spaces, whereas they only prove convergence in $L^{1}$. We also prove $C^{\infty}$ convergence in compact subsets where our solution is positive. Finally, in terms of producing an approximation scheme, it would seem to be preferable to be working with Stefan approximations as opposed to porous medium approximations, as the Stefan problem is much closer to being linear, and, in fact, we have established some estimates about the convergence of the free boundaries of our approximating problems.

Inwon Kim and her coauthors have recently studied the regularity of viscosity solutions to the Hele-Shaw problem (without a mushy region). (See [Ki], JK], and CJK.) Making various regularity assumptions including that the free boundary is a Lipschitz graph, Kim is able to show that locally the free boundary is $C^{1}$ and the solution is also locally $C^{1}$ in a neighborhood of the free boundary. Kim and her coauthors address certain short time regularity questions in time and space for various singular initial configurations of the free boundary in the plane. Determining whether the notion of weak solution or the notion of viscosity solution leads to a larger class of solutions (or if there is no inclusion) is currently an important and open problem.

The classical version of the $m$-approximating problem which we use is given as follows:

1.1. Definition ( $m$-approximating problem). Let $\alpha_{m}(s):=m(s-1)_{+}$. We let $u^{(m)}$ denote the solution of the following partial differential equation, where we write $V^{(m)}$ as an abbreviation for $\alpha_{m}\left(u^{(m)}\right)$,

$$
u_{t}^{(m)}(x, t)=\Delta V^{(m)}(x, t), \quad(x, t) \in D^{c} \times(0,+\infty) \cap\left\{u^{(m)}>1\right\},
$$

with boundary data given by

$$
\begin{aligned}
& u^{(m)}(x, 0)=u_{I}(x), \quad x \in D^{c}, \\
& V^{(m)}(x, t)=p(x, t), \quad(x, t) \in \partial D \times(0,+\infty),
\end{aligned}
$$

and with the free boundary condition

$$
\left(-\nabla_{x} V^{(m)},\left(1-u_{I}\right)\right) \cdot \nu=0, \quad(x, t) \in \partial\left\{u^{(m)}>1\right\} .
$$

Here $\nu$ is the outer $(n+1)$-dimensional normal to the set $\left\{u^{(m)}>1\right\}$, and this free boundary condition will be satisfied when the free boundary is smooth. The weak formulation we give at the beginning of the next section will not require any regularity assumptions on the free boundary or on the initial data. For a fixed $m>0$, we call the free boundary problem determined by the equations above the m-approximating problem. We will assume

$$
\begin{aligned}
& 0<p(x, t) \in C^{2, \alpha}(\partial D \times(0,+\infty)), \\
& D \text { is a bounded set with } \partial D \in C^{2, \alpha} .
\end{aligned}
$$

The following assumption will lead to major simplifications in the proofs in this weak setting, but we will be able to establish most of the results even without it:

$$
p(x, t) \text { is nondecreasing in time for each fixed } x .
$$

(PNDT) 


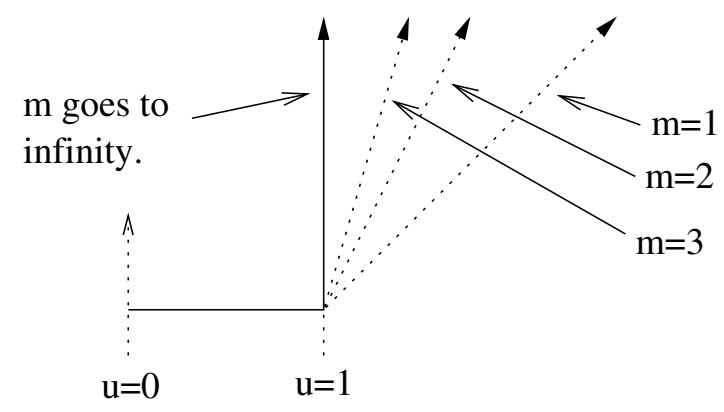

FiguRE 1

Of course, for $p(x, t)=p(x)$ the assumption (PNDT) is automatically satisfied. For convenience, we will define

$$
M:=\|p\|_{L^{\infty}(\partial D \times(0,+\infty))} .
$$

The boundedness in time assumed here is obviously not necessary, as we can simply consider $p$ on the set $\partial D \times(0, T]$ to get a bound. Now in terms of the positivity assumption on $p(x, t)$, we note that $p(x, t) \equiv 0$ leads to a trivial case. There will be no evolution at all. To see this fact extend each $u^{(m)}$ by 1 across all of $D$. In short, the positivity of $p(x, t)$ is driving the evolution.

In this formulation $u^{(m)}$ is energy (or enthalpy), and $V^{(m)}=m\left(u^{(m)}-1\right)_{+}$ is temperature. (In this paper $\alpha_{m}(s)$ will always denote $m(s-1)_{+}$, and $V^{(m)}$ will always denote $\alpha_{m}\left(u^{(m)}\right)$.) Equation (1.2) comes from conservation of energy. Basically, as $m$ increases, the diffusion happens faster. Competing with this increase in diffusion is the fact that the boundary data for $u^{(m)}$ on $\partial D$ is decreasing down to 1 . As $m \rightarrow \infty$ we have convergence of our operators as shown in Figure 1, which is typical for Mesa problems.

We show that as $m \rightarrow \infty$, the $u^{(m)}$ converge pointwise to a limit $0 \leq u^{(\infty)} \leq 1$, and the $V^{(m)}$ converge pointwise to a bounded function $V^{(\infty)}(x, t)$. If we add the assumption (PNDT), then the convergence becomes monotone. Furthermore, the function $V^{(\infty)}$ is identically zero in $\left\{u^{(\infty)}<1\right\}$ and positive and harmonic for fixed $t$ in the component of the set $\left\{u^{(\infty)}=1\right\}$ which contains $D$. (On sets where $\left\{u^{(\infty)}=1\right\}$ which are isolated from $D$ we will have $V^{(\infty)} \equiv 0$; see Remark [3.1.) Finally, the pair $\left(u^{(\infty)}, V^{(\infty)}\right)$ is a weak solution to our Hele-Shaw problem with a mushy region which we state formally in Definition 2.7. Note that this definition yields a natural generalization of the weak formulation of the notion of solution to the Hele-Shaw problem used by DiBenedetto and Friedman (see DF]). Moreover, after invoking a result of Bouillet, we will be able to say that solutions of our formulation are unique, and hence the Mesa limiting procedure we use gives an effective method of constructing the solution. Finally, we address the regularity of the free boundary.

The paper is arranged as follows. In section 2 we introduce our notion of weak solutions to the approximating problem, show some qualitative properties of these solutions, derive the existence of the limits (for now in weak-* $L^{\infty}$ ), and give some trivial bounds on these limits. Most of the results in this section draw from the maximum principle and from the papers $[\mathrm{AK},[\mathrm{K}]$, and [DK]. Section 3 gives a simple counterexample which motivates the definition of the free boundary and of 
the diffusive region, and also motivates our nondegeneracy hypothesis (5.3) which is assumed in the later sections. In section 4 we derive some monotonicity properties of our sequences and limits when we assume (PNDT), and as consequences we show that our convergence is actually monotone, that the limit $V^{(\infty)}$ is continuous in space, and that even without (PNDT) the diffusive sets are nested with respect to time. In section 5 we derive the Cacciopoli estimates for our solutions, and we use these estimates to show that the Baiocchi transforms of all of the approximating problems are solutions of simple obstacle problems. As a further consequence we get pointwise convergence of our approximators. In section 6 we return to the general case and show that at every single moment in time, the wet region is the noncontact set for a simple obstacle problem and so its regularity is already well understood. (See [C] and [B] .)

\section{THE WEAK FORMULATIONS}

For our $m$-approximating problem, we need an appropriate weak formulation, which we give here.

2.1. Definition (Weak solutions of the $m$-approximating problem). The nonnegative function $u^{(m)}(x, t) \in L_{l o c}^{1}$ is a weak solution of the $m$-approximating problem if

$$
\limsup _{|x| \rightarrow \infty} u^{(m)}(x, t)=0
$$

and for any $\varphi \in C^{\infty}\left(\mathbb{R}^{n} \times[0, \infty)\right)$, such that $\varphi \equiv 0$ on $\partial D \times[0, \infty)$, and $\varphi(x, t) \rightarrow 0$ as either $t \rightarrow \infty$ or $|x| \rightarrow \infty$ we have

$$
\begin{aligned}
& \int_{D^{c}} \int_{0}^{\infty} \varphi_{t}(x, t) u^{(m)}(x, t) d t d x+\int_{D^{c}} \int_{0}^{\infty} \Delta_{x} \varphi(x, t) V^{(m)}(x, t) d t d x \\
& =\int_{\partial D} \int_{0}^{\infty} \frac{\partial \varphi}{\partial \nu}(x, t) p(x, t) d t d \mathcal{H}^{n-1}(x)-\int_{D^{c}} \varphi(x, 0) u_{I}(x) d x .
\end{aligned}
$$

We observe that the traces of $u^{(m)}$ and $V^{(m)}=m\left[u^{(m)}-1\right]_{+}$on the boundaries of our domain will be well defined by the work of Korten even if we only assume that we are dealing with local solutions and that the initial trace is between zero and one. (See $[\mathrm{K}]$, which adapts the work of Dahlberg and Kenig. Furthermore, we observe that the free boundary condition for the classical formulation (i.e. Equation (1.4) ) will be satisfied whenever the functions and sets are sufficiently smooth.

2.2. Remark. Equation (2.1) is simply a technical assumption to prevent unbounded solutions on $D^{c}$ with no free boundary. As an example of what needs to be ruled out, consider $p \equiv 1$ with $D$ as the unit disk in $\mathbb{R}^{2}$, and $V^{m}(x)=1+\log |x|$.

For strictly local situations, it may be helpful to note that if $\varphi \in C_{0}^{\infty}(\Omega)$ where $\Omega \Subset D^{c} \times(0,+\infty)$, then we have

$$
\begin{aligned}
& \int_{D^{c}} \int_{0}^{\infty} \varphi_{t}(x, t) u^{(m)}(x, t) d t d x \\
= & -\int_{D^{c}} \int_{0}^{\infty} \Delta_{x} \varphi(x, t) V^{(m)}(x, t) d t d x \\
= & \int_{D^{c}} \int_{0}^{\infty} \nabla_{x} \varphi(x, t) \cdot \nabla_{x} V^{(m)}(x, t) d t d x .
\end{aligned}
$$




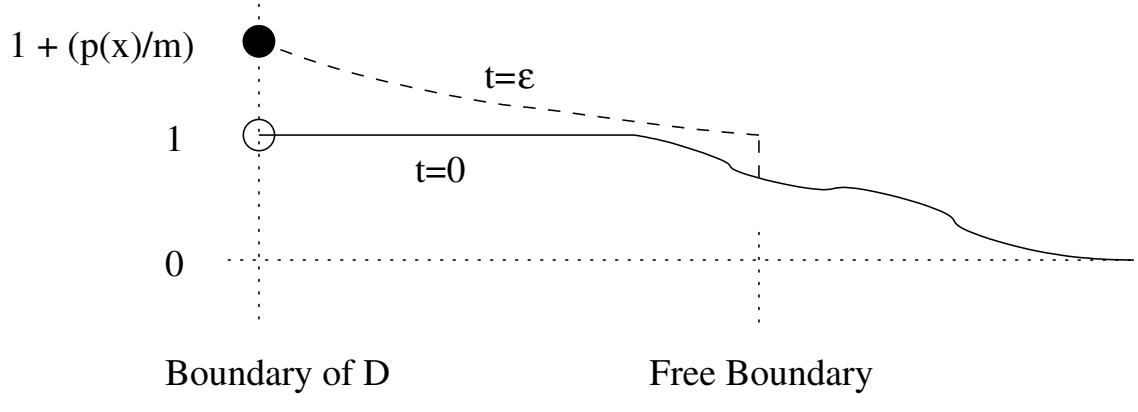

FIGURE 2

The last integration by parts requires that we invoke the known regularity theory and energy estimates for solutions of Equation (1.2). See Lemma 1.2 of [AK.

Since we want to discuss regularity of functions in Sobolev spaces, we need to fix ideas about which representative we will use. Although the "Lebesgue point" representative where points are filled in by taking limits of averages over balls with radii going to zero is the most common procedure, we will use a slightly different approach which exploits the fact that the functions $u^{(m)}(x, t)$ are continuous in $t$ once $u^{(m)}$ jumps above its initial value. (The "jump" theorem follows this definition.)

2.3. Definition (Representative). For $t>0$, we set

$$
\tilde{u}^{(m)}(x, t):=\lim _{s \uparrow t} u^{(m)}(x, s) \text { for a.e. } x .
$$

For economy of notation we will not bother to relabel any of our $u^{(m)}$ 's, but always understand that we are using $\tilde{u}^{(m)}$ as our representative for $u^{(m)}$ in all pointwise matters.

The $u^{(m)}$ are not continuous across the free boundary, and in fact, we have the following theorem:

2.4. Theorem $\left(u^{(m)}\right.$ must jump). Almost every $x \in \mathbb{R}^{n}$ has the property: If $u^{(m)}(x, t)<1$ for a.e. $t \in(a, b)$, then $u^{(m)}(x, t)$ is constant a.e. in $(a, b)$.

On the other hand, $m\left(u^{(m)}-1\right)_{+}$will be continuous across the free boundary. (See $[\mathrm{K}$, which verifies the required assumptions of $[\mathrm{DB}]$ for continuity.) For a fixed $m$ we now have the picture of $u^{(m)}$ viewed from the side (see Figure 2).

Proof. First observe that if the segment $\{(x, t): t \in(a, b)\}$ lies in the interior of the set where $u^{(m)}(x, t)<1$, then the equation easily implies that $u^{(m)}(x, t)$ must be constant. On the other hand, we cannot assume that the set where $u^{(m)}(x, t)$ is less than one is open. It suffices to show this result for almost every $x \in B_{R}(0)$ where $R>0$, and we can assume that $a$ and $b$ are rational. Let $E:=\left\{x \in B_{R}(0)\right.$ : $u^{(m)}(x, t)<1$ for a.e. $\left.t \in(a, b)\right\}$, and assume that $|E|>0$.

Consider a nonnegative, even $\eta \in C_{0}^{\infty}(\mathbb{R})$ with $\int_{\mathbb{R}} \eta(x) d x=1$. By taking a countable number of translates and dilates of $\eta$ and differentiating, we can produce a sequence $\left\{\eta_{j}^{\prime}(t)\right\}$ of $C^{\infty}$ functions which are compactly supported in $(a, b)$ and which enjoy the property:

$$
f \in L^{2}(a, b) \text { and } \int_{a}^{b} \eta_{j}^{\prime}(t) f(t) d t=0 \quad \forall j \text { implies } f \equiv C \text { on }(a, b) .
$$


So, to complete the proof it suffices to show that for each $j$ we have

$$
\int_{a}^{b} \eta_{j}^{\prime}(t) u^{(m)}(x, t) d t=0 \text { for a.e. } x \in E \text {. }
$$

Fix $j$, and let $\varphi(x) \in C_{0}^{\infty}\left(B_{9 / 10}\right)$ be nonnegative, radial, and have $\int_{B_{1}} \varphi(x) d x=1$.

Let $0<r<1$. For $x \in B_{R}, \frac{1}{r^{n}} \varphi\left(\frac{x-y}{r}\right) \eta_{j}(t)$ is a $C^{\infty}$ function of $(y, t)$ and is compactly supported in $B_{R+1} \times(a, b)$. So, if $x \in B_{R}$, then we can use Equation (2.2) to get (recall that $\alpha_{m}(s):=m(s-1)_{+}$)

$$
\begin{array}{r}
\int_{a}^{b} \int_{B_{R+1}} u^{(m)}(y, t) \frac{1}{r^{n}} \varphi\left(\frac{x-y}{r}\right) \eta_{j}^{\prime}(t) d y d t \\
=\int_{a}^{b} \int_{B_{R+1}} \alpha_{m}\left(u^{(m)}(y, t)\right) \frac{1}{r^{n+2}} \Delta \varphi\left(\frac{x-y}{r}\right) \eta_{j}(t) d y d t .
\end{array}
$$

For $y \in B_{R+1}$, set

$$
h(y):=\int_{a}^{b} \eta_{j}^{\prime}(t) u^{(m)}(y, t) d t
$$

and

$$
h_{r}(y):=\int_{B_{R+1}} h(y) \frac{1}{r^{n}} \varphi\left(\frac{x-y}{r}\right) d y .
$$

Then $h, h_{r} \in L^{2}\left(B_{R+1}\right)$, and as $r \rightarrow 0, h_{r} \rightarrow h$ a.e. and in $L^{2}\left(B_{R+1}\right)$. We need to show that $h(y)=0$ a.e. in $E$.

Set

$$
k(y):=\int_{a}^{b} \eta_{j}(t) \alpha_{m}\left(u^{(m)}(y, t)\right) d t
$$

and

$$
k_{r}(y):=\int_{B_{R+1}} k(y) \frac{1}{r^{n}} \varphi\left(\frac{x-y}{r}\right) d y .
$$

Since $u \in L_{l o c}^{2}$, energy estimates imply that $k(y) \in W^{1,2}\left(B_{R+1}\right)$. We also have $k_{r} \rightarrow k$ a.e. and in $L^{2}\left(B_{R+1}\right)$.

Now with these definitions, Equation (2.5) can be written simply as $h_{r}(x)=$ $\Delta k_{r}(x)$, for $x \in B_{R+1}, 0<r<1$. Now let $\psi \in C^{\infty}\left(\mathbb{R}^{n}\right)$ be compactly supported in $B_{R}$. Then

$$
\begin{aligned}
\int_{B_{R}} h(x) \psi(x) d x & =\lim _{r \rightarrow 0} \int_{B_{R}} h_{r}(x) \psi(x) d x \\
& =\lim _{r \rightarrow 0} \int_{B_{R}} \Delta k_{r}(x) \psi(x) d x \\
& =\lim _{r \rightarrow 0} \int_{B_{R}} k_{r}(x) \Delta \psi(x) d x \\
& =\int_{B_{R}} k(x) \Delta \psi(x) d x .
\end{aligned}
$$

Thus $k \in W^{1,2}\left(B_{R}\right)$ is a weak solution of $\Delta k=h$ in $B_{R}$. By Theorem 8.8 of [GT], we conclude that $k \in W^{2,2}\left(\Omega^{\prime}\right)$ for any $\Omega^{\prime} \Subset B_{R}$, and $\Delta k=h$ a.e. in $B_{R}$. By 
Lemma 7.7 of [GT], $\Delta k=0$ a.e. on any set where $k$ is constant, and in particular this must hold on $E$ where $k \equiv 0$. Therefore $h=0$ a.e. on $E$ as desired.

By using the boundedness of the data along with the maximum principle that solutions of Equations (2.2) and (2.1) enjoy, we can conclude that the solutions are bounded, and therefore by elementary functional analysis we can conclude that the following limit

$$
u^{(\infty)}:=\lim _{m \rightarrow \infty} u^{(m)}
$$

exists weak- $* L^{\infty}$ along a subsequence of $m$ in the entire domain. Again by using the maximum principle it is immediate that

$$
0 \leq u^{(\infty)} \leq 1
$$

In fact, by using the maximum principle again, we can assert that

$$
0 \leq u^{(m)} \leq 1+\frac{M}{m} \quad \text { or } \quad 0 \leq m\left(u^{(m)}-1\right)_{+} \leq M
$$

and we stress that $M$ is independent of $m$ and $t$. Because of this fact, we can take a further subsequence to ensure that $V^{(m)}$ has a limit $V^{(\infty)}$ in the weak-* $L^{\infty}$ topology of the entire domain.

2.5. Remark. By combining Equation (2.12) with Theorem 2.4 we can conclude that the essential range of $u^{(m)}(x, \cdot)$ is a subset of $\left\{u_{I}(x)\right\} \cup[1,1+M / m]$.

Next, for $\varphi \in H_{0}^{1}(\Omega)$ we have:

$$
\begin{aligned}
\int_{0}^{+\infty} \int_{D^{c}} \nabla_{x} V^{(m)} \cdot \nabla_{x} \varphi d x d t & =\int_{0}^{+\infty} \int_{D^{c}} u^{(m)} \varphi_{t} d x d t \\
& \rightarrow \int_{0}^{+\infty} \int_{D^{c}} u^{(\infty)} \varphi_{t} d x d t
\end{aligned}
$$

Since $u^{(\infty)} \in L^{\infty}(\Omega) \subset L^{2}(\Omega)$, we have

$$
u_{t}^{(\infty)} \in H^{-1}(\Omega) .
$$

(We have assumed that $\Omega$ is compactly contained within $D^{c} \times(0, \infty)$.) We summarize with the following lemma whose proof is now obtained trivially by using the weak-* $L^{\infty}$ compactness we have and taking the limits as $m \rightarrow \infty$ in the weak formulation of the $m$-approximating problem.

2.6. Lemma (Limits solve the limiting problem). Under our assumptions as above, we have

$$
0 \leq V^{(\infty)} \leq M
$$

and the pair $\left(u^{(\infty)}, V^{(\infty)}\right)$ satisfies

$$
\begin{aligned}
& \int_{D^{c}} \int_{0}^{\infty} \varphi_{t}(x, t) u^{(\infty)}(x, t) d t d x+\int_{D^{c}} \int_{0}^{\infty} \Delta_{x} \varphi(x, t) V^{(\infty)}(x, t) d t d x \\
= & \int_{\partial D} \int_{0}^{\infty} \frac{\partial \varphi}{\partial \nu}(x, t) p(x, t) d t d \mathcal{H}^{n-1}(x)-\int_{D^{c}} \varphi(x, 0) u_{I}(x) d x
\end{aligned}
$$

for any $\varphi \in C^{\infty}\left(\mathbb{R}^{n} \times[0, \infty)\right)$, such that $\varphi \equiv 0$ on $\partial D \times[0, \infty)$, and $\varphi(x, t) \rightarrow 0$ as either $t \rightarrow \infty$ or $|x| \rightarrow \infty$. 
For the next definition we let

$$
\alpha_{\infty}(s):= \begin{cases}0 & s<1 \\ {[0,+\infty)} & s \geq 1 .\end{cases}
$$

2.7. Definition (Hele-Shaw with mushy region). Any pair $\left(u^{(\infty)}, V^{(\infty)}\right)$ which satisfies $V^{(\infty)} \in \alpha_{\infty}\left(u^{(\infty)}\right)$ a.e., which satisfies Equation (2.14), and which satisfies Equation (2.15) for any $\varphi$ as in the lemma above will be called a weak solution of the Hele-Shaw problem with boundary data $p(x, t)$ and initial data $u_{I}(x)$.

Now in order to show that our pair $\left(u^{(\infty)}, V^{(\infty)}\right)$ is a weak solution of the HeleShaw problem, it remains to show that $V^{(\infty)} \in \alpha_{\infty}\left(u^{(\infty)}\right)$ a.e. Because we only have weak-* convergence so far, this result is nontrivial, and we will prove it in the fourth section as an immediate consequence of pointwise convergence almost everywhere. Granting that result temporarily, we get uniqueness of our limits.

2.8. Theorem (Uniqueness among all solutions). The solution of the limiting problem of Lemma 2.6 is unique, so all solutions of the Hele-Shaw problem as given in Definition 2.7 are recoverable via the Mesa limit process we have introduced.

Proof. This theorem is an immediate application of the uniqueness theorem in section 3 of $\mathrm{BO}$ when combined with Lemma 2.6 and Theorem 5.5 .

\section{EXTREMAL TYPES OF BOUNDARY DATA}

This section discusses two extremal cases for the boundary data. Consideration of these cases is important in order to make appropriate definitions of the free boundary. Technical problems arise if the initial data $u_{I}$ is not bounded away from 1 , or if the lateral boundary data $p(x, t)$ vanishes for any time, $t_{0}$.

Based on the assumptions we have so far, the function $V^{(\infty)}(x, t)$ will not be continuous in time in general. To show this, we will assume for the sake of this example that $u_{I}$ is a continuous function. We will also assume in this example that the set $D$ is connected. The trouble appears in certain cases where the set

$$
\mathcal{W}:=D \cup\left\{x \in \mathbb{R}^{n}: u_{I}(x)=1\right\}
$$

is disconnected. If $K$ is a component of the set $\left\{u_{I}=1\right\}$ and $K$ is a positive distance away from $D$, then the $u^{(m)}$ and therefore the $u^{(\infty)}$ should not evolve on this set of $x$ until the free boundary comes into contact with it. Essentially, the "patch" $K$ will not "see" the input of the slot until the component of the set $\left\{u^{(m)} \geq 1\right\}$ which surrounds $D$ connects to it. (See Figure 3.)

3.1. Remark $\left(V^{(\infty)}\right.$ is not always continuous). To produce a situation where $V^{(\infty)}$ must be discontinuous in time, simply consider the radially symmetric situation where $D=B_{1}$ and $u_{I}(r)$ is taken such that it is identically one on the set $3 \leq r \leq 5$, but smaller outside of it. When what we want to call the free boundary reaches $r=3$, then it will instantaneously jump to $r=5$, and this leads to an immediate jump in the height of $V^{(\infty)}$.

The next extremal example comes when $p\left(x, t_{0}\right)$ vanishes for all $x \in \partial D$. If we take $u_{I} \equiv 0$, and do everything with radial symmetry to ensure the existence of classical solutions, then it is clear that at time $t_{0}$, because $V^{(\infty)}\left(\cdot, t_{0}\right)$ is harmonic and has zero boundary data we must have $V^{(\infty)} \equiv 0$. This situation is distinct from 


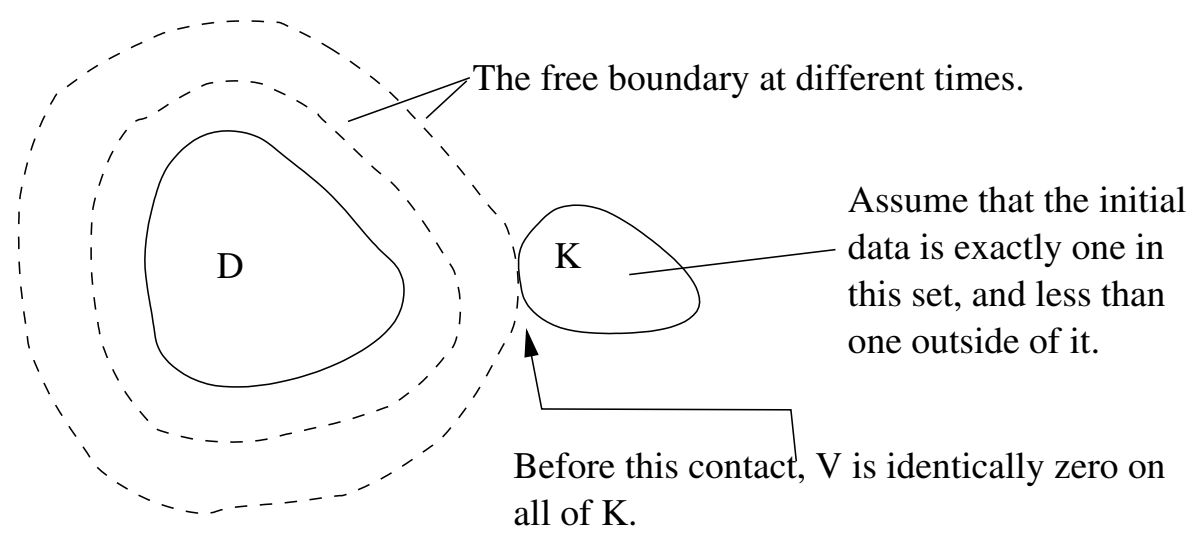

FiguRE 3

the parabolic approximating problems where the set $\left\{u^{(m)}>1\right\}$ is identical to the set $\left\{V^{(m)}>0\right\}$.

With these two examples in mind we make the following definitions:

3.2. Definition (Defining the free boundary). We start by defining the diffusive region at time $t$ for the $m$-approximating problem and the limiting Hele-Shaw problem to be respectively,

$$
\begin{aligned}
& A^{(m)}(t):=\left\{x \in D^{c}: u^{(m)}(x, t) \geq 1\right\}, \\
& A^{(\infty)}(t):=\left\{x \in D^{c}: u^{(\infty)}(x, t)=1\right\} .
\end{aligned}
$$

Now we simply define the free boundary at time $t$ to be $F B^{(m)}(t):=\partial A^{(m)}(t) \backslash \partial D$, or $F B^{(\infty)}(t):=\partial A^{(\infty)}(t) \backslash \partial D$, according to which problem we are considering. (Here " $\partial K$ " denotes the topological boundary of $K$.)

Observe that because of the first radially symmetric example given above where $V^{(\infty)}$ is discontinuous in time, it is also clear that the free boundary cannot be expected to vary continuously in time.

We need some notation before proving the next theorem. We set

$$
\begin{aligned}
& t_{m}(x):=\inf \left\{t: V^{(m)}(x, t)>0\right\}, \\
& t_{\infty}(x):=\inf \left\{t: V^{(\infty)}(x, t)>0\right\},
\end{aligned}
$$

and

$$
\begin{aligned}
& \tau_{m}(x):=\inf \left\{t: u^{(m)}(x, t) \geq 1\right\}, \\
& \tau_{\infty}(x):=\inf \left\{t: u^{(\infty)}(x, t)=1\right\} .
\end{aligned}
$$

By considering Figure 3, it is clear that in general $t_{m}(x)$ does not have to equal $\tau_{m}(x)$, and similarly with $t_{\infty}(x)$ and $\tau_{\infty}(x)$. In the figure, they would differ on the set $K$. For $x \in K$ we have $\tau_{m}(x)=\tau_{\infty}(x)=0$, while $t_{m}(x)$ would be the positive time when the moving part of the boundary of $\left\{u^{(m)}>1\right\}$ crosses $K$, and $t_{\infty}(x)$ would be the positive time when the moving part of the boundary of $\left\{u^{(\infty)}=1\right\}$ crosses $K$. Now due to the one-phase nature of this problem it is intuitive that the diffusive regions should be nested in time. We prove that here. 
3.3. Theorem (Diffusive regions are nested in time). Almost every $t_{0}$ has the property that for a.e. $t>t_{0}$, we have

$$
\mathcal{L}^{n}\left\{A^{(\infty)}\left(t_{0}\right) \backslash A^{(\infty)}(t)\right\}=0 .
$$

Proof. We will simply show that $u_{t}^{(\infty)}$ is a nonnegative measure, which implies this result. First observe that because weak-* $L^{\infty}$ convergence is stronger than convergence in the sense of distributions, we have

$$
\begin{aligned}
& V^{(m)} \stackrel{\mathcal{D}^{\prime}}{\rightarrow} V^{(\infty)}, \\
& u^{(m)} \stackrel{\mathcal{D}^{\prime}}{\rightarrow} u^{(\infty)} .
\end{aligned}
$$

In fact, since differentiation is a continuous operator on the space of distributions, we even have

$$
\begin{aligned}
\Delta V^{(m)} & \stackrel{\mathcal{D}^{\prime}}{\rightarrow} \Delta V^{(\infty)}, \\
u_{t}^{(m)} & \stackrel{\mathcal{D}^{\prime}}{\rightarrow} u_{t}^{(\infty)} .
\end{aligned}
$$

Note also, that since $\left(u^{(m)}-1\right)_{+}$converges to zero uniformly it follows that

$$
\frac{\partial}{\partial t}\left(u^{(m)}-1\right)_{+} \stackrel{\mathcal{D}^{\prime}}{\rightarrow} 0 .
$$

Using this last fact we conclude that $\Delta V^{(m)}-\frac{\partial}{\partial t}\left(u^{(m)}-1\right)_{+}$also converges to $\Delta V^{(\infty)}$. From Equation (2.2) we know that $\Delta V^{(m)}=u_{t}^{(m)}$, so we have

$$
\begin{aligned}
\Delta V^{(m)}-\frac{\partial}{\partial t}\left(u^{(m)}-1\right)_{+} & =u_{t}^{(m)}-\frac{\partial}{\partial t}\left(u^{(m)}-1\right)_{+} \\
& =\left(u^{(m)}-\left(u^{(m)}-1\right)_{+}\right)_{t} \\
& =: \lambda_{t}^{(m)} .
\end{aligned}
$$

Now observe that the functions $\lambda^{(m)}$ are monotone increasing functions in $t$. (In fact they pick up only the free boundary jump. See [DK].) Thus we can conclude that the $\lambda_{t}^{(m)}$ are nonnegative measures, and these measures converge in the sense of distributions to $u_{t}^{(\infty)}$.

3.4. Remark ("Diffusive" versus "Wet"). The reader may observe that "Wet" may be a more physically appropriate name for what we are calling the diffusive region in the Hele-Shaw problem. On the other hand, "wet" might more properly describe any region where $u^{(\infty)}(x, t)>0$, so we will stick to what is already appropriate in the approximating problems, and certainly appropriate when within the context of quasi-static Stefan problems; namely, we call the set $\left\{0<u^{(\infty)}<1\right\}$ the mushy region, and the set $\left\{u^{(\infty)}=1\right\}$ the diffusive region.

3.5. Corollary (Representation of $\left.u^{(\infty)}\right)$. There is an increasing set-valued function of $t$ which we call $Q(t)$ such that $u^{(\infty)}(x, t)$ admits the representation for almost every $(x, t)$ :

$$
u^{(\infty)}(x, t)=\chi_{Q(t)}(x)+u_{I}(x) \chi_{Q(t)^{c}}(x) .
$$


Furthermore, $u^{(\infty)}(x, t)$ is the pointwise limit of the functions $u^{(m)}(x, t)$ almost everywhere, and $Q(t)$ can be chosen to be equal to the set $\left\{x \in \mathbb{R}^{n}: \tau_{\infty}(x)<t\right\}$. $(Q(t)$ is increasing means in the sense of set inclusion.)

Proof. By Theorem 3.3, we know that $u^{(\infty)}(x, t)$ is an increasing function of $t$ and so by using Theorem 3.3 along with Equation (2.11) we can conclude that $u_{I}(x) \leq$ $u^{(\infty)}(x, t) \leq 1$. So, to prove Equation (3.9) it suffices to show that $u^{(\infty)}(x, \cdot)$ does not attain values strictly between $u_{I}(x)$ and 1 for a.e. $x$. Indeed, by combining the previous theorem with Remark 2.5 we see that the limit

$$
\lim _{m \rightarrow \infty} u^{(m)}\left(x_{0}, t_{0}\right)
$$

exists for almost every $\left(x_{0}, t_{0}\right) \in \mathbb{R}^{n+1}$ and is either equal to 1 or to $u_{I}\left(x_{0}\right)$. At that point it is a simple exercise to show that this pointwise limit coincides with the weak-* $L^{\infty}$ limit almost everywhere.

In terms of the "choice" of $Q(t)$, it is clear that the only $x$ where there can be a choice is on the (possibly empty) set $\left\{x \in \mathbb{R}^{n}: u_{I}(x)=1\right\}$. Examination of the definition of $Q(t)$ combined with the monotonicity of $u^{(\infty)}(x, t)$ in $t$ makes it clear that $Q(t):=\left\{x \in \mathbb{R}^{n}: \tau_{\infty}(x)<t\right\}$ will suffice. (Note that an equally good choice for $Q(t)$ would be to take $Q(t):=\left\{x \in \mathbb{R}^{n}: t_{\infty}(x)<t\right\}$.)

\section{Convergence When $p$ IS NONDECREASing}

For this section we will assume that $p$ is nondecreasing as a function of time. We will have this as a standing assumption until the last corollary of this section. We start with a lemma which is a simple consequence of Lemma 4.2 in [ $\mathrm{K}$.

4.1. Lemma (Monotonicity of $\left.u^{(m)}\right)$. For each $m>0$ and any $x \in D^{c}$ we have that $u^{(m)}(x, t)$ is a nondecreasing function of time.

Proof. If $u^{(m)}(x, t)<1$, then this follows immediately from Lemma 4.2 of $[\underline{\mathrm{K}}]$. As a consequence of this fact, we can already say that the $A^{(m)}(t)$ are nested nondecreasing sets. Now we consider the set $S:=\left\{(x, t): u^{(m)}(x, t)>1\right\}$. By observing that $u^{(m)}$ satisfies the heat equation within $S$ and invoking standard parabolic regularity theory, we can conclude that $u^{(m)}(x, t)$ will assume the boundary values $1+\frac{p(x, t)}{m}$ on $\partial D$ continuously, except possibly at the initial corner. By Corollary 1.3 of $\mathrm{mK}$ the function $\left(u^{(m)}-1\right)_{+}$is continuous within $D^{c}$, so we can extend $u^{(m)}$ to be 1 on the rest of the parabolic boundary of $S$ in a continuous fashion, except again at $t=0$ where this set meets $\partial D$. Now we consider the function

$$
U(x, t):=u^{(m)}(x, t+\epsilon)-u^{(m)}(x, t) .
$$

Within $S$, this function satisfies the heat equation. On $\partial S \cap \partial D$, we have $U(x, t) \equiv 0$. On the rest of the parabolic boundary of $S$ we have $U(x, t)=u^{(m)}(x, t+\epsilon)-1 \geq 0$. In the corner where $U(x, t)$ is discontinuous, it stays bounded between zero and $1+\frac{M}{m}$, and hence by the weak maximum principle, $U(x, t)$ is nonnegative in all of $D$. Hence

$$
\frac{u^{(m)}(x, t+\epsilon)-u^{(m)}(x, t)}{\epsilon}
$$

is nonnegative, and the result follows by taking $\epsilon \downarrow 0$. 
Now without data on the slot which "competes" with the increasing diffusivity (or if the functions solve the equation on all of $\mathbb{R}^{n}$ ), it is immediate by rescaling that the diffusive regions (the $A^{(m)}(t)$ ) must be nondecreasing with $m$. In our case, however, we need to produce an appropriate barrier.

4.2. Theorem (Temperature increases with $m$ ). If $m<k$, then

$$
m\left[u^{(m)}-1\right]_{+} \leq k\left[u^{(k)}-1\right]_{+} .
$$

As a trivial consequence we can say that the $A^{(m)}(t)$ are nested: $m<k$ implies $A^{(m)}(t) \subset A^{(k)}(t)$.

Proof. We make the following definition:

$$
v^{(k)}(x, t):= \begin{cases}u^{(m)}(x, t) & \text { if } u^{(m)}(x, t)<1 \\ 1+\frac{m}{k}\left(u^{(m)}(x, t)-1\right) & \text { if } u^{(m)}(x, t) \geq 1\end{cases}
$$

and claim that $v^{(k)}(x, t)$ is a subsolution of the $k$-approximating problem. Indeed, this follows very quickly from the following two easily verifiable statements:

(1) The set where $v^{(k)}>1$ is identical to the set where $u^{(m)}>1$.

(2) $k\left(v^{(k)}-1\right)_{+} \equiv m\left(u^{(m)}-1\right)_{+}$.

(Only the definition of $v^{(k)}$ is needed to verify these statements!) By the first observation the free boundaries of $v^{(k)}$ and $u^{(m)}$ are identical in time and space, so the speed of these boundaries at every point is also identical. On the other hand, the second observation quickly leads to the conclusion that the function $v^{(k)}$ satisfies the free boundary condition and the boundary value condition on the slot for the $k$-approximating problem exactly. Finally, we simply compute:

$$
\begin{aligned}
v_{t}^{(k)}(x, t) & \leq u_{t}^{(m)}(x, t) \\
& =m \Delta\left(u^{(m)}(x, t)-1\right)_{+} \\
& =k \Delta\left(v^{(k)}(x, t)-1\right)_{+},
\end{aligned}
$$

which shows that $v^{(k)}$ is a local subsolution to the $k$-approximating problem, and therefore $v^{(k)}(x, t) \leq u^{(k)}(x, t)$. (In the first inequality above we have used Lemma 4.1) We now have the simple consequence

$$
k\left[u^{(k)}-1\right]_{+} \geq k\left[v^{(k)}-1\right]_{+} \equiv m\left[u^{(m)}-1\right]_{+},
$$

which is the monotonicity we require.

4.3. Corollary (Pointwise convergence of the temperature). The sequence of functions $\left\{V^{(m)}\right\}$ converges pointwise almost everywhere to $V^{(\infty)}$. In particular, the limiting function $V^{(\infty)}$ is unique. (No subsequence is ever needed.)

Proof. By the last theorem combined with the estimates in place already, at each point we have a bounded increasing sequence.

4.4. Remark (Uniqueness of limits of subsequences). Although we had originally needed subsequences to be sure that our $u^{(m)}$ would converge in weak-* $L^{\infty}$, the last result makes it clear that $u^{(m)}$ will converge both pointwise and weak-* $L^{\infty}$ to $u^{(\infty)}$ without the need to extract a subsequence. 
4.5. Corollary $\left(V^{(\infty)}\right.$ is harmonic within the diffusive region). The spatial Laplacian of $V^{(\infty)}$ is zero for $x$ in the interior of $A(t)$. As a consequence, if $\partial D \in C^{k, \alpha}$ where $k \geq 2$, then $V^{(\infty)}$ attaches to the slot $D$ in a $C^{k, \alpha}$ fashion.

\section{Energy estimates and Baiocchi transforms}

For $m=1,2, \ldots, \infty$ we apply the Baiocchi transformation to $V^{(m)}(x, t)$ and define:

$$
W^{(m)}(x, t):=\int_{0}^{t} V^{(m)}(x, s) d s .
$$

Since we know the $V^{(m)}$ 's are bounded by the maximum principle, it follows that $W^{(m)}(\cdot, t) \in L^{\infty}\left(D^{c}\right)$. Observe also that by the one-phase nature of our approximating problem it is clear that the set $\left\{W^{(m)}>0\right\}$ is identical to the set $\left\{V^{(m)}>0\right\}$. Now we will show that $W^{(m)}(\cdot, t)$ belongs to $H_{l o c}^{1}\left(D^{c}\right)$ for almost every $t$, and then that $W^{(m)}(x, t)$ is a weak solution of the following obstacle problem in almost every time slice $t=T$ :

$$
\left.\begin{array}{l}
0 \leq W^{(m)}(x, T), \\
W^{(m)}(x, T)=\int_{0}^{T} p(x, s) d s \quad \text { for } x \in \partial D, \\
\Delta_{x} W^{(m)}(x, T)=\chi_{\left\{W^{(m)}(x, T)>0\right\}}(x)\left(u^{(m)}(x, T)-u_{I}(x)\right) .
\end{array}\right\}
$$

After that we will be able to invoke stability results for the obstacle problem due to Blank (see [B] $)$ to establish stronger convergence results for the $u^{(m)}$ and the $V^{(m)}$. We will also be able to invoke the regularity results for the obstacle problem due to Caffarelli, Kinderlehrer, Nirenberg, and Blank.

We will not assume (PNDT) in this section, but we will assume the following nondegeneracy condition henceforth:

$$
u_{I}(x) \leq \lambda<1
$$

( $u_{I}$ satisfying Equation (5.3) will be referred to as nondegenerate initial data.) We start by stating some simple trace results. Basically, we need to adapt Equation (2.2) to some situations with slightly different test functions.

5.1. Lemma (First Trace result). If $\psi(x) \in C^{\infty}$ is supported in the interior of $D^{c}$, then the following formula holds for a.e. $T$ for finite $m$ :

$$
\begin{aligned}
& \int_{D^{c}} \int_{0}^{T}\left[\Delta_{x} \psi(x)\right] \alpha_{m}\left(u^{(m)}(x, t)\right) d t d x \\
& =\int_{D^{c}} \psi(x)\left[u^{(m)}(x, T)-u_{I}(x)\right] d x
\end{aligned}
$$

Proof. We make the following definition:

$$
\varphi(x, t):=\left\{\begin{array}{rr}
\psi(x) & t \leq T \\
\Theta(x, t) & t>T
\end{array}\right.
$$

where $\Theta(x, t)$ is chosen to ensure that $\varphi(x, t)$ is a permissible test function for our $m$-approximating problem. In particular, we need $\varphi(x, t) \in C^{\infty}$ and we need it to converge to zero as $t \rightarrow \infty$. Neither requirement poses any difficulty. 
By using the trace result of [AK] (see Theorem 1.1 of $\mathrm{AK}$ ) our functions $u^{(m)}(x, t)$ solve our $m$-approximating problem starting at time $T$ with initial data $u^{(m)}(x, T)$ for almost every $T$, and so (for those $T$ ) we can use $\Theta(x, t)$ as the test function in Equation (2.2) to obtain:

$$
\begin{aligned}
& \int_{D^{c}} \int_{T}^{\infty} \Theta_{t}(x, t) u^{(m)}(x, t) d t d x \\
+ & \int_{D^{c}} \int_{T}^{\infty} \Delta_{x} \Theta(x, t) \alpha_{m}\left(u^{(m)}(x, t)\right) d t d x \\
= & \int_{D^{c}} \psi(x) u^{(m)}(x, T) d x .
\end{aligned}
$$

(Note that $\Theta(x, T)=\psi(x)$ since we have required that $\varphi(x, t)$ be smooth.) Now by subtracting this equation from what we have when we substitute the function $\varphi(x, t)$ defined in Equation (5.5) into Equation (2.2) we get Equation (5.4) immediately.

The proof of the following result is almost identical to the proof of the trace result above, so we omit it.

5.2. Lemma (Second Trace result). For a.e. $t_{0}, t_{1}$ such that $0 \leq t_{0}<t_{1}<\infty$, and for $\varphi \in C^{\infty}\left(\mathbb{R}^{n} \times\left[t_{0}, t_{1}\right]\right)$, which satisfies $\varphi \equiv 0$ on an open set containing $D \times\left[t_{0}, t_{1}\right]$, we have

$$
\begin{aligned}
& \int_{D^{c}} \int_{t_{0}}^{t_{1}} \varphi_{t}(x, t) u^{(m)}(x, t) d t d x+\int_{D^{c}} \int_{t_{0}}^{t_{1}} \Delta_{x} \varphi(x, t) \alpha_{m}\left(u^{(m)}(x, t)\right) d t d x \\
& =\int_{D^{c}}\left(\left.\left[\varphi(x, s) u^{(m)}(x, s)\right]\right|_{s=t_{0}} ^{s=t_{1}}\right) d x
\end{aligned}
$$

5.3. Theorem (Spatial Regularity for $V^{(m)}(\cdot, t)$ ). For almost every time $t$, $V^{(m)}(\cdot, t) \in H_{l o c}^{1}\left(D^{c}\right)$.

Proof. We take $\psi(x):=\phi(|x| / r), \phi \in C^{\infty}$ with $2 r<R$, and $\phi \equiv 1$ in $[0,1]$ and $\phi \equiv 0$ in $[2, \infty)$. We can assume that $\psi$ satisfies $\left\|D^{\alpha} \psi\right\|_{L^{\infty}} \leq \frac{C}{r^{|\alpha|}}$, and we will assume that $B_{2 r} \subset D^{c}$. Because we need more smoothness than we can currently assume about our functions, we will take $\alpha_{m}^{\epsilon}$ to be a smooth approximation to $\alpha_{m}$ so that the solutions to the corresponding equation are $C^{\infty}$ and approximate the solutions to the problem with the old $\alpha_{m}$ 's. In fact, we will have the following convergences:

$$
\begin{aligned}
\alpha_{m}^{\epsilon}\left(u^{(m, \epsilon)}\right) & \rightarrow \alpha_{m}\left(u^{(m)}\right) & & \text { a.e., } \\
\nabla_{(x, t)} \alpha_{m}^{\epsilon}\left(u^{(m, \epsilon)}\right) & \rightarrow \nabla_{(x, t)} \alpha_{m}\left(u^{(m)}\right) & & \text { weakly in } L^{2} \\
u^{(m, \epsilon)} & \rightarrow u^{(m)} & & \text { weakly in } L^{2} .
\end{aligned}
$$

These convergences follow as in $\mathrm{AK}$. Now, by using the formula from Lemma 5.2 (it still holds in this setting) and by taking $\varphi^{\epsilon}(x, t):=\psi(x)^{2} \alpha_{m}^{\epsilon}\left(u^{(m, \epsilon)}(x, t)\right.$ ), we 
get

$$
\begin{aligned}
& \int_{B_{2 r}} \psi(x)^{2} \int_{t_{0}}^{t_{1}} \frac{\partial}{\partial t}\left[\alpha_{m}^{\epsilon}\left(u^{(m, \epsilon)}(x, t)\right)\right] u^{(m, \epsilon)}(x, t) d t d x \\
& +\int_{B_{2 r}} \int_{t_{0}}^{t_{1}} \Delta_{x}\left[\psi(x)^{2} \alpha_{m}^{\epsilon}\left(u^{(m, \epsilon)}(x, t)\right)\right] \alpha_{m}^{\epsilon}\left(u^{(m, \epsilon)}(x, t)\right) d t d x \\
& =\int_{B_{2 r}}\left(\left.\left[\psi(x)^{2} \alpha_{m}^{\epsilon}\left(u^{(m, \epsilon)}(x, s)\right) u^{(m, \epsilon)}(x, s)\right]\right|_{s=t_{0}} ^{s=t_{1}}\right) d x
\end{aligned}
$$

So, that means

$$
\begin{aligned}
& \int_{B_{2 r} \cap\left\{u^{(m, \epsilon)}>1\right\}} \int_{t_{0}}^{t_{1}} \psi^{2} \frac{\partial}{\partial t}\left(\frac{1}{2 m} \alpha_{m}^{\epsilon}\left(u^{(m, \epsilon)}\right)^{2}+\alpha_{m}^{\epsilon}\left(u^{(m, \epsilon)}\right)\right) d t d x \\
& -\int_{B_{2 r}} \int_{t_{0}}^{t_{1}} \nabla_{x}\left[\psi^{2} \alpha_{m}^{\epsilon}\left(u^{(m, \epsilon)}\right)\right] \bullet \nabla_{x}\left[\alpha_{m}^{\epsilon}\left(u^{(m, \epsilon)}\right)\right] d t d x \\
& =\int_{B_{2 r}}\left(\left.\left[\psi(x)^{2} \alpha_{m}^{\epsilon}\left(u^{(m, \epsilon)}(x, s)\right) u^{(m, \epsilon)}(x, s)\right]\right|_{s=t_{0}} ^{s=t_{1}}\right) d x .
\end{aligned}
$$

Now let

$$
\begin{gathered}
I_{1}:=\int_{B_{2 r} \cap\left\{u^{(m, \epsilon)}>1\right\}} \int_{t_{0}}^{t_{1}} \psi^{2} \frac{\partial}{\partial t}\left(\frac{1}{2 m} \alpha_{m}^{\epsilon}\left(u^{(m, \epsilon)}\right)^{2}+\alpha_{m}^{\epsilon}\left(u^{(m, \epsilon)}\right)\right) d t d x \\
I_{2}:=\int_{B_{2 r}} \int_{t_{0}}^{t_{1}} \nabla_{x}\left[\psi^{2} \alpha_{m}^{\epsilon}\left(u^{(m, \epsilon)}\right)\right] \bullet \nabla_{x}\left[\alpha_{m}^{\epsilon}\left(u^{(m, \epsilon)}\right)\right] d t d x
\end{gathered}
$$

and

$$
I_{3}:=\int_{B_{2 r}}\left(\left.\left[\psi(x)^{2} \alpha_{m}^{\epsilon}\left(u^{(m, \epsilon)}(x, s)\right)\right]\right|_{s=t_{0}} ^{s=t_{1}}\right) d x .
$$

Then we have the following estimates:

$$
\begin{aligned}
\left|I_{1}\right| & \leq\left|\int_{B_{2 r}}\left[\frac{1}{2 m}\left(\alpha_{m}^{\epsilon}\left(u^{(m, \epsilon)}\right)\right)^{2}+\alpha_{m}^{\epsilon}\left(u^{(m, \epsilon)}\right)\right]\right|_{s=t_{0}}^{s=t_{1}} d x \mid \\
& \leq\left(\frac{1}{2 m} M^{2}+M\right)\left|B_{2 r}\right|
\end{aligned}
$$

and

$$
\left|I_{3}\right| \leq M\left|B_{2 r}\right|
$$

Now by using these estimates and the inequality relating the geometric and arithmetic means, we arrive at

$$
\int_{B_{r}} \int_{t_{0}}^{t_{1}}\left|\nabla_{x} \alpha_{m}^{\epsilon}\left(u^{(m, \epsilon)}\right)\right|^{2} d t d x \leq C M r^{n}\left(1+M\left(t_{1}-t_{0}\right) r^{-2}\right)
$$

once $m$ is sufficiently large. Thus, for any ball compactly contained within $D^{c}$ we have a uniform $H^{1}$ bound on our sequence $\left\{\alpha_{m}^{\epsilon}\left(u^{(m, \epsilon)}\right)\right\}$. By letting $\epsilon \downarrow 0$ and by using the lower semicontinuity of the Dirichlet integral under weak convergence, we get a uniform $H^{1}$ bound on our sequence $\left\{\alpha_{m}\left(u^{(m)}\right)\right\}$. 
5.4. Theorem (Spatial Regularity for $\left.W^{(m)}(\cdot, t)\right)$. For all $t>0$, we have $W^{(m)}(\cdot, t)$ $\in H_{l o c}^{1}\left(D^{c}\right)$. Further, for any $K$ compactly contained within $D^{c}$ the bound on $\left\|W^{(m)}(\cdot, t)\right\|_{H^{1}(K)}$ is uniform in $m$.

Proof. Let $K$ be a compact subset of $D^{c}$. Since $W^{(m)}(\cdot, t) \leq M t$, we have $W^{(m)}(\cdot, t)$ $\in L^{\infty}(K) \subset L^{2}(K)$. It remains to show that $\frac{\partial W^{(m)}}{\partial x_{i}}(\cdot, t) \in L^{2}(K)$.

Let $\varphi \in C_{0}^{1}(K)$ and estimate.

$$
\begin{aligned}
\left(\frac{\partial W^{(m)}}{\partial x_{i}}, \varphi\right) & =-\int_{K} W^{(m)}(x, t) \frac{\partial \varphi}{\partial x_{i}}(x) d x \\
& =-\int_{K} \int_{0}^{t} V^{(m)}(x, s) \frac{\partial \varphi}{\partial x_{i}}(x) d s d x \\
& =-\int_{K} \int_{0}^{t} \alpha_{m}\left(u^{(m)}(x, s)\right) \frac{\partial \varphi}{\partial x_{i}}(x) d s d x \\
& =\int_{K} \int_{0}^{t} \frac{\partial}{\partial x_{i}}\left(\alpha_{m}\left(u^{(m)}(x, s)\right)\right) \varphi(x) d s d x \\
& =\int_{K} \varphi(x)\left[\int_{0}^{t} \frac{\partial}{\partial x_{i}}\left(\alpha_{m}\left(u^{(m)}(x, s)\right)\right) d s\right] d x
\end{aligned}
$$

Now, by using Minkowski's integral inequality and the Cauchy-Schwarz Inequality we have

$$
\begin{aligned}
& \left|\int_{K} \varphi(x)\left[\int_{0}^{t} \frac{\partial}{\partial x_{i}}\left(\alpha_{m}\left(u^{(m)}(x, s)\right)\right) d s\right] d x\right| \\
& \leq\left|\int_{K} \varphi(x)^{2} d x\right|^{1 / 2}\left|\int_{K}\left(\int_{0}^{t} \frac{\partial}{\partial x_{i}}\left(\alpha_{m}\left(u^{(m)}(x, s)\right)\right) d s\right)^{2} d x\right|^{1 / 2} \\
& \leq\|\varphi\|_{L^{2}(K)} \int_{0}^{t}\left|\int_{K}\left(\frac{\partial}{\partial x_{i}}\left(\alpha_{m}\left(u^{(m)}(x, s)\right)\right)\right)^{2} d x\right|^{1 / 2} d s \\
& \leq\|\varphi\|_{L^{2}(K)}\left|\int_{0}^{t} \int_{K}\left(\frac{\partial}{\partial x_{i}}\left(\alpha_{m}\left(u^{(m)}(x, s)\right)\right)\right)^{2} d x d s\right|^{1 / 2} \cdot\left[\int_{0}^{t} d s\right]^{1 / 2} \\
& \leq t^{1 / 2}\|\varphi\|_{L^{2}(K)}\left\|\nabla_{x} \alpha_{m}\left(u^{(m)}(x, s)\right)\right\|_{L^{2}(K \times(0, t))} \\
& \leq C t^{1 / 2}\|\varphi\|_{L^{2}(K)},
\end{aligned}
$$

where the last constant is independent of $m$ by using Equation (5.11).

At this point, for finite $m$, Equation (5.2) follows from our first trace result, Lemma 5.1, and so we can start using obstacle problem results to gain more information about our solutions to the original problem. We recall our obstacle problem formulation:

$$
\begin{aligned}
& 0 \leq W^{(m)}(x, T) \\
& W^{(m)}(x, T)=\int_{0}^{T} p(x, s) d s \quad \text { for } x \in \partial D \\
& \Delta_{x} W^{(m)}(x, T)=\chi_{\left\{W^{(m)}(x, T)>0\right\}}(x)\left(u^{(m)}(x, T)-u_{I}(x)\right) .
\end{aligned}
$$


Now we still need to establish the existence of a contact set. Indeed, we can solve the obstacle problem on the unbounded domain $B_{1}^{c}$ with $C(|x|-1)_{+}^{2}$ and then there is no contact set or hope of using the weak maximum principle. What saves us is the $L^{\infty}$ bound on $W^{(m)}$ obtained from the $L^{\infty}$ bound on $V^{(m)}$. With this bound and with the nonnegativity of $V^{(m)}$, it is a simple matter to use the function $C\left|x-x_{0}\right|^{2}$ combined with the weak maximum principle to show that not only does the set $\left\{W^{(m)}(x, T) \equiv 0\right\}$ exist, but in fact the set $\left\{W^{(m)}(x, T)>0\right\}$ must have compact support. This will follow in the same way that the standard nondegeneracy theorems for the obstacle problem are proven. (See [Bl] or [C].)

The function $u^{(m)}$ is bounded by $1+M / m$ by the maximum principle, so we can now bound the $W^{(m)}(x, T)$ from below by defining $Y^{(m)}(x, T)$ to be the solution of the following obstacle problem:

$$
\begin{aligned}
& 0 \leq Y^{(m)}(x, T) \\
& Y^{(m)}(x, T)=\int_{0}^{T} p(x, s) d s \quad \text { for } x \in \partial D \\
& \Delta_{x} Y^{(m)}(x, T)=\chi_{\left\{Y_{(m)}^{(m, T)>0\}}\right.}(x)\left(1-u_{I}(x)+\frac{M}{m}\right) .
\end{aligned}
$$

We will soon show that $W^{(\infty)} \equiv Y^{(\infty)}$ where we define $Y^{(\infty)}$ to be the solution of the obstacle problem:

$$
\left.\begin{array}{l}
0 \leq Y^{(\infty)}(x, T), \\
Y^{(\infty)}(x, T)=\int_{0}^{T} p(x, s) d s \quad \text { for } x \in \partial D, \\
\Delta_{x} Y^{(\infty)}(x, T)=\chi_{\left\{Y_{(\infty)}(x, T)>0\right\}}(x)\left(1-u_{I}(x)\right) .
\end{array}\right\}
$$

In the meantime, however, we simply note the inequalities:

$$
Y^{(m)} \leq W^{(m)} \leq Y^{(\infty)}
$$

$$
Y^{(m)} \leq Y^{(m+1)}
$$

which all follow by using the weak maximum principle. (See [Bl, Theorem 2.7] for example.) We introduce notation for $m=1,2, \ldots, \infty$ :

$$
\Omega^{(m)}(t):=\left\{x \in D^{c}: Y^{(m)}(x, t)>0\right\}
$$

and observe that Equation (5.14) immediately implies

$$
\Omega^{(m)}(t) \subset A^{(m)}(t) \subset \Omega^{(\infty)}(t)
$$

(recall the definition of $A^{(m)}(t)$ given in Equation (3.2)), and Equation (5.15) yields

$$
\Omega^{(m)}(t) \subset \Omega^{(m+1)}(t)
$$

Now by redoing the proof of Theorem 4.1 of [B] for an exterior domain we obtain the following estimate:

$$
\mathcal{L}^{n}\left\{\Omega^{(\infty)}(t) \backslash \Omega^{(m)}(t)\right\} \leq \frac{C t}{m}
$$

Now if $A \Delta B$ denotes the symmetric difference of the sets $A$ and $B$, then since $m<n$ implies

$$
A^{(m)}(t) \Delta A^{(n)}(t) \subset \Omega^{(\infty)}(t) \backslash \Omega^{(m)}(t),
$$


we conclude that the characteristic functions of the $A^{(m)}(t)$ 's form a Cauchy sequence in $L^{1}\left(D^{c}\right)$. Indeed the same estimates show that (with the obvious abuse of notation) the characteristic functions of the $A^{(m)}$ 's form a Cauchy sequence in $L^{1}\left(D^{c} \times[0, T]\right)$ for any fixed positive $T$. Taking a subsequence we get convergence almost everywhere. By combining this convergence with Theorem 2.4 along with Equation (2.12) we easily obtain the following theorem.

5.5. Theorem $\left(u^{(m)}\right.$ 's converge pointwise). $u^{(m)}$ 's converge pointwise almost everywhere to $u^{(\infty)}$.

5.6. Corollary $\left(u^{(\infty)}\right.$ must jump). Almost every $x \in \mathbb{R}^{n}$ has the property: If $u^{(\infty)}(x, t)<1$ for a.e. $t \in(a, b)$, then $u^{(\infty)}(x, t)$ is constant a.e. in $(a, b)$.

This fact now leads to the desired equations for the Baiocchi transform of the limiting problem:

5.7. Theorem $\left(W^{(\infty)}\right.$ solves the limiting obstacle problem). $W^{(\infty)}$ is the unique solution of the obstacle problem given in (5.13).

Proof. The convergence is good enough now to verify the trace result of Lemma 5.1 for the case $m=\infty$. Nonnegativity of $W^{(\infty)}$ is already known, and since by Theorem 5.4 we can take a subsequence of the $W^{(m)}(\cdot, T)$ 's to converge weakly to $W^{(\infty)}(\cdot, T)$ in $H^{1}(K)$ for any $K \Subset D^{c}$ we know that $W^{(\infty)}(\cdot, T) \in H_{l o c}^{1}\left(D^{c}\right)$. Uniqueness follows from the uniqueness of solutions to the obstacle problem with given boundary data.

Now we get the following corollary by standard obstacle problem regularity statements. (See [C] or [B] or the references within.)

5.8. Corollary (Obstacle problem regularity). For any $T>0$ we have $W^{(\infty)}(\cdot, T) \in$ $C^{1,1}\left(D^{c}\right)$.

5.9. Remark (Nontriviality and nondegeneracy). Due to the competition between the decreasing data on $\partial D$ and the increasing diffusivity of our $m$-approximating problems, we need to rule out two trivial cases. The possibilities that we need to exclude are:

(1) The possibility that the free boundary moves to infinity as soon as $t>0$.

(2) The possibility that the free boundary never moves.

Both of these issues can now be resolved using standard results for the obstacle problem along with Equation (5.14). In fact these problems can be solved without reference to the obstacle problem, but simply by constructing an appropriate sequence of barrier functions and appealing to the weak maximum principle. Although this construction is potentially useful in situations where a change of variables does not lead to the obstacle problem, it is much more complicated.

\section{Regularity of the Free Boundary in SPACE}

We turn now to spatial regularity results for the free boundary. To begin we need a definition:

6.1. Definition (Minimum diameter). The minimum diameter of a set $S \subset \mathbb{R}^{n}$ (denoted "m.d. $(S)$ ") is the infimum of the distances between parallel hyperplanes enclosing $S$. 
6.2. Theorem (Regularity of the free boundary in space). Assume $u_{I}$ is continuous, nondegenerate initial data. Then there is a modulus of continuity $\sigma$ which depends on $\lambda, n$, and the modulus of continuity of $u_{I}$ such that at almost any time $t$ (indeed every time $t_{0}$ where Equation (5.2) is valid) and for any free boundary point $\left(x_{0}, t_{0}\right)$ contained in the interior of $D^{c}$ we have either (with $B_{r}(x)$ denoting the (spatial) ball centered at $x$ with radius $r$ )

$$
\text { m.d. }\left(B_{r}\left(x_{0}\right) \cap A^{(\infty)}(t)^{c}\right) \leq r \sigma(r) \quad \text { for all } r \leq 1
$$

(so that the nondiffusive region is "cusp-like") or

$$
\lim _{r \rightarrow 0} \frac{\left|B_{r}\left(x_{0}\right) \cap A^{(\infty)}(t)\right|}{\left|B_{r}\left(x_{0}\right)\right|}=\frac{1}{2}
$$

in which case we will say that $\left(x_{0}, t_{0}\right)$ is a regular point of the free boundary.

Proof. Simply use Equation (5.2) together with the results from [B].

6.3. Theorem (Better regularity). Assume the hypotheses of the previous theorem and assume that $\left(x_{0}, t_{0}\right)$ is a regular point of the free boundary. Then the free boundary intersected with $\left\{t=t_{0}\right\}$ will be Reifenberg vanishing near $x_{0}$. (For a definition of Reifenberg vanishing, see B1].) If $u_{I}$ is Dini continuous, then the free boundary will be $C^{1}$ (in space) near $x_{0}$, and if $u_{I} \in C^{k, \alpha}$, then the free boundary will be $C^{k+1, \alpha}$ (in space) near $x_{0}$.

Proof. Again, just combine Equation (5.2) with the results from [B].

6.4. Remark (Clarification). When we say "near" $x_{0}$ in the theorem above we mean near in space only. In other words, we are specifically talking about the free boundary restricted to the time slice $t=t_{0}$.

At this point we need to call attention to our assumptions that $u_{I}$ is continuous and nondegenerate. Indeed it is well known that there are examples of "persistent corners" in Hele-Shaw problems. (See [KLV] for example.) Our theorem does not contradict this fact. Our spatial regularity theorem says nothing if $u_{I}$ is discontinuous, or if the set $\left\{u_{I}=1\right\} \cap D^{c}$ is nonempty. On the other hand, our assumptions do not rule out "focusing" or changes in topology of the diffusive region, so it is certainly nontrivial that corners do not arise in this setting. Consider a case where $u_{I}$ is very close to zero except for an $\epsilon$ neighborhood of an annulus which contains the slot. In this annulus assume that $u_{I}$ is extremely close to one. (The epsilon neighborhood is needed to make $u_{I}$ continuous.) In this case, the diffusive region will make its way around the annulus in each direction very quickly, but expand slowly into the region inside and outside of the annulus. Thus, it will meet itself on the other side of the annulus long before it fills in the interior. (See Figure 4.)

We wish now to improve the last set of regularity results by extending them from almost every time to every time. Indeed, this prevents corners from arising even as "transitions" from the situations which are permissible for sets of time with positive measure. (Consider for example the sets $S(t):=\{(x, y): x y<t-1\}$ for the interval $t \in[0,2]$. By the results of the last section, it would still be possible for $S(t)$ to be the diffusive region for a Hele-Shaw flow, since the corner only occurs when $t$ belongs to the zero measure set $\{1\}$.) Of course we still need the standing assumption that $u_{I}$ is nondegenerate initial data (see Equation (5.3)). We also note that any corners which would "persist" as in [KLV] are already impossible, but the following results will eliminate corners with any angle from ever appearing. 


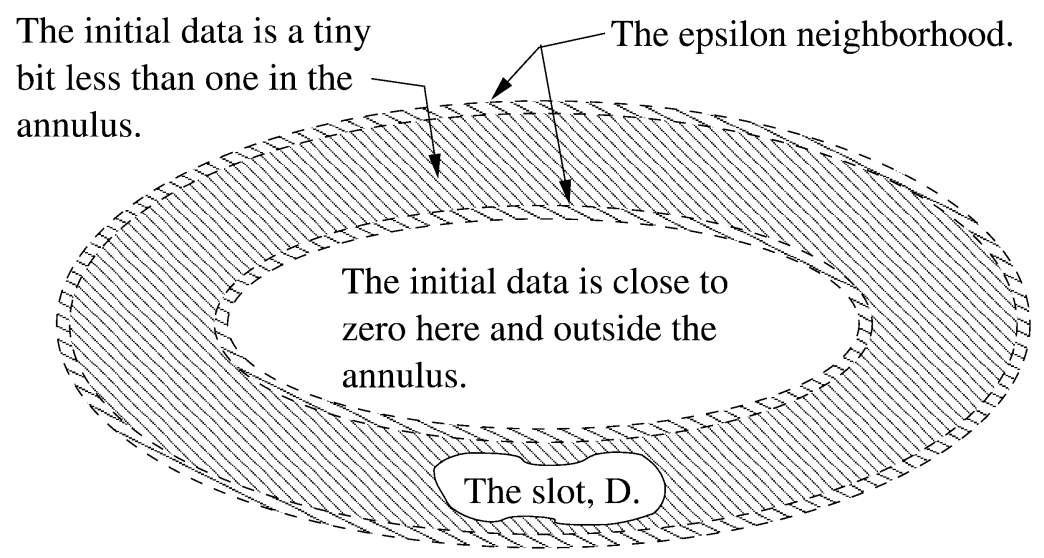

FIGURE 4

6.5. Theorem (Measure of the diffusive region). There exists a constant $C=$ $C(n, \alpha, \partial D)$ such that if $0 \leq t-s \leq 1$ and $t \leq T$, then

$$
|A(t) \backslash A(s)| \leq C\|p\|_{C^{2, \alpha}(\partial D \times[0, T])}\left(\frac{t-s}{1-\lambda}\right) .
$$

(For ease of notation, we will from this point forward use $A(t)$ to denote $A^{(\infty)}(t)$ and similarly with $W(x, t)$ and $V(x, t)$. We recall that $A^{(\infty)}(t)$ is the diffusive region at time $t$, and for $S \subset \mathbb{R}^{n}$, we are letting $|S|$ denote the Lebesgue $n$-dimensional measure of $S$.)

Proof. We adapt the proof of Theorem 4.1 of [B] to the current setting. Fix $t$ and $s$. Because the diffusive regions are nested, and because Equation (5.2) holds for almost every time, without loss of generality we can assume that it holds for both $t$ and $s$. Let $L:=A(t) \backslash A(s)$, let $\Psi(x):=W(x, t)-W(x, s)$ and observe that $\Delta \Psi(x)=\chi_{L}\left(1-u_{I}\right) \geq 0$, and

$$
\begin{array}{ll}
\Psi(x)=0 & x \in F B(t), \\
\Psi(x)=\int_{s}^{t} p(x, r) d r & x \in \partial D .
\end{array}
$$

From this fact and by the weak maximum principle, it follows that

$$
0 \leq W(x, t)-W(x, s) \leq(t-s)\|p\|_{L^{\infty}(\partial D \times[0, T])}
$$

for all $x \in A(t)$. (Nonnegativity is actually a consequence of the increasing boundary data for $W$.)

Observe that $W(x, t)-W(x, s)$ is harmonic within $A(s)$ so that

$$
\begin{aligned}
0 & =\int_{A(s)} \Delta(W(x, t)-W(x, s)) d x \\
& =\int_{\partial D} \frac{\partial}{\partial \nu}(W(x, t)-W(x, s)) d \mathcal{H}^{n-1}-\int_{F B(s)} \frac{\partial}{\partial \nu}(W(x, t)-W(x, s)) d \mathcal{H}^{n-1} \\
& =\int_{\partial D} \frac{\partial}{\partial \nu}(W(x, t)-W(x, s)) d \mathcal{H}^{n-1}-\int_{F B(s)} \frac{\partial}{\partial \nu} W(x, t) d \mathcal{H}^{n-1},
\end{aligned}
$$

where the last equality follows from Corollary 5.8. Now by using boundary regularity for harmonic functions combined with the second part of Equation (6.4) we 
get

$$
\left|\int_{\partial D} \frac{\partial}{\partial \nu}(W(x, t)-W(x, s)) d \mathcal{H}^{n-1}\right| \leq C(n, \alpha, \partial D)(t-s)\|p\|_{C^{2, \alpha}(\partial D)} .
$$

By combining this fact with the last computation, we conclude that

$$
\left|\int_{F B(s)} \frac{\partial}{\partial \nu} W(x, t) d \mathcal{H}^{n-1}\right| \leq C(n, \alpha, \partial D)(t-s)\|p\|_{C^{2, \alpha}(\partial D)} .
$$

On the other hand, by using Corollary 5.8 in the last equality below we have

$$
\begin{aligned}
(1-\lambda)|L| & \leq \int_{L}\left(1-u_{I}\right) d x \\
& =\int_{L} \Delta W(x, t) d x \\
& =\int_{\partial L} \frac{\partial}{\partial \nu} W(x, t) d \mathcal{H}^{n-1} \\
& =\int_{F B(s)} \frac{\partial}{\partial \nu} W(x, t) d \mathcal{H}^{n-1},
\end{aligned}
$$

which we can combine with Equation (6.7) to give us what we need.

6.6. Corollary (Continuity in $L^{p}$ ). Under the assumptions made at the beginning of this section, the map from $t$ to the function

$$
\chi_{\{W(x, t)>0\}}\left(1-u_{I}\right)
$$

is a continuous function from $\mathbb{R}$ into $L^{p}\left(D^{c}\right)$ for $1 \leq p<\infty$.

6.7. Corollary (Spatial regularity for every time). All of the results of Theorems 6.2 and 6.3 hold for every time.

Proof. It suffices to show that Equation (5.2) holds for all time. Fix $\tilde{t}>0$ and let $t_{n} \rightarrow \tilde{t}$ with $t_{n}$ chosen so that Equation (5.2) holds at each $t_{n}$. Now take a ball, $B_{R}$, which is large enough to contain $A(\tilde{t})$ in its interior, and let $\Omega:=B_{R} \backslash D$. For ease of notation, let $\bar{P}(x, t):=\int_{0}^{t} p(x, s) d s$. For each $n$ we let $w_{n}(x)$ solve the boundary value problem

$$
\begin{array}{ll}
\Delta w_{n}(x)=\chi_{A\left(t_{n}\right)}\left(1-u_{I}\right) & \text { in } \Omega, \\
w_{n}(x)=\bar{P}\left(x, t_{n}\right) & \text { on } \partial D, \\
w_{n}(x)=0 & \text { on } \partial B_{R} .
\end{array}
$$

By standard uniqueness results, $w_{n}(x) \equiv W\left(x, t_{n}\right)$, as they satisfy the same boundary value problem. By standard elliptic regularity theory, since the boundary data on $\partial D$ will converge to $\bar{P}(x, \tilde{t})$ and by the last corollary the right-hand side of the equation will converge in $L^{p}(\Omega)$ to $\chi_{A(\tilde{t})}\left(1-u_{I}\right)$, we can conclude that $w_{n}$ will converge to a function $\tilde{w}$ which satisfies

$$
\begin{array}{ll}
\Delta \tilde{w}(x)=\chi_{A(\tilde{t})}\left(1-u_{I}\right) & \text { in } \Omega, \\
\tilde{w}(x)=P(x, \tilde{t}) & \text { on } \partial D, \\
\tilde{w}(x)=0 & \text { on } \partial B_{R} .
\end{array}
$$

On the other hand, $w_{n}(x)=W\left(x, t_{n}\right)$ converges to $W(x, \tilde{t})$ by the continuity of $W$ in time. 


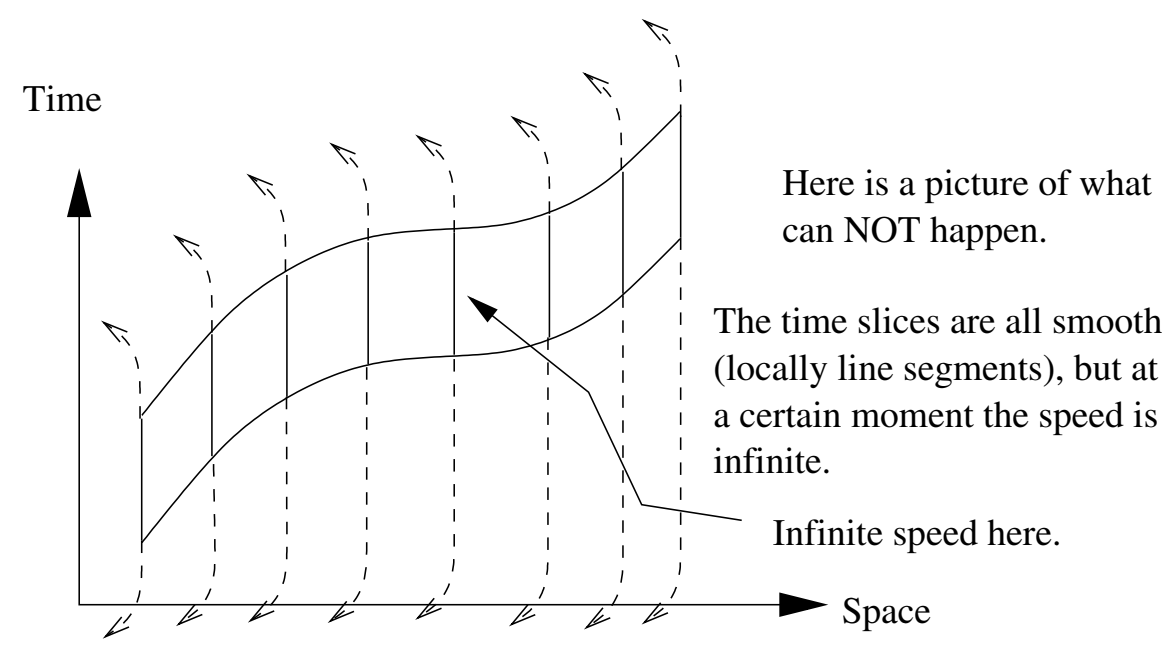

FigURE 5

\section{Partial Regularity in time}

In this section we will establish that if the function $u_{I}$ is Hölder continuous, then sufficiently near (in time and space) any spatially smooth free boundary point we have Lipschitz regularity in time. This local Lipschitz result cannot be made global, as it is well known that due to focussing effects, for example, the best that can be hoped for is Hölder continuity in time. It does show, however, that smooth fronts must have finite speed or lose their smoothness. (See Figure 5.)

In the course of proving this result, we will also prove that in regions where $\partial A(t)$ is regular, the approximating free boundaries (i.e. $\partial A^{(m)}(t)$ ) converge uniformly to $\partial A(t)$. We will also be able to find a quantitative estimate giving the rate of convergence.

In terms of understanding this section, one should keep in mind that at the outset there is still no control in time of the free boundary. For this reason, it is hard to create suitable test functions for the parabolic problem. On the other hand, after the problem is transformed and we are looking at the obstacle problem in each time slice, we can then make a barrier in any given slice and then we can use standard regularity estimates since we $d o$ have spatial regularity.

In order to proceed we need to more carefully quantify our notion of a spatially smooth free boundary. We let $d_{\mathcal{H}}(A, B)$ denote the Hausdorff distance between the sets $A$ and $B$. We will call $\left(x_{0}, t_{0}\right)$ an $\eta$-good free boundary point at the level $r$ if there exists a spatial hyperplane $H\left(x_{0}\right)$ such that

$$
d_{\mathcal{H}}\left(H\left(x_{0}\right) \cap B_{r}\left(x_{0}\right), F B\left(t_{0}\right) \cap B_{r}\left(x_{0}\right)\right) \leq \eta .
$$

For ease of notation, we will use $\mathcal{G}(\eta, r)$ to denote the set of all $\eta$-good free boundary points at the level $r$. Note that $\eta$-good at all levels less than or equal to a given $r_{0}$ is the same as Reifenberg flat.

7.1. Theorem (Stability of spatially smooth free boundaries). Assume $0 \leq u_{I} \leq$ $\lambda<1$, and that $u_{I}$ is a continuous function. 
(1) Assume $\left(x_{0}, t_{0}\right) \in \mathcal{G}(\eta, 1)$, and $\eta \leq 1 / 10$ is sufficiently small. There exists an $\epsilon>0$ and an $r_{0}>0$ such that all free boundary points within the cylinder $B_{r_{0} / 2}\left(x_{0}\right) \times\left[t_{0}, t_{0}+\epsilon\right]$ belong to $\mathcal{G}(2 r \eta, r / 4)$ for all $r<r_{0}$.

(2) Let $0<\gamma<1$, and assume that $B_{1}\left(x_{0}\right) \cap D=\varnothing$. Then there exists an $r_{0}>0$ and a $\rho \in(0,1)$ such that if for some $r \leq r_{0}$ we have $\left(x_{0}, t_{0}\right) \in \mathcal{G}(\gamma r, r)$, then all free boundary points within the cylinder $B_{r \rho}\left(x_{0}\right) \times\left[t_{0}, t_{0}+\rho\right]$ belong to $\mathcal{G}(\gamma r \rho, r \rho / 2)$.

Proof. This follows as in Lemma 5.3 of $[\mathrm{Bl}]$.

Note that the last results show that the regular points of the free boundary form a relatively open subset of the free boundary.

7.2. Remark (Regular free boundary points are all good). It follows from [B] that as long as we assume that $u_{I}$ is continuous we know that the free boundary is Reifenberg vanishing near regular points. Because of this fact, for any $1 / 10 \geq \eta>0$, and any level $L>0$, if we have a regular free boundary point, $(\tilde{x}, \tilde{t})$, then we can rescale to assume that $(\tilde{x}, \tilde{t}) \in \mathcal{G}(\eta, L)$.

So by rescaling, by the last remark, and by the theorem above, once we are at a regular free boundary point we can assume that we are in a neighborhood where all of the points are as good as we like. Furthermore, if we assume that $u_{I} \in C^{\alpha}$, then we can use standard regularity theory (see [B] or [C]) to conclude that the free boundary is the graph of a $C^{1, \alpha}$ function, and as close to a hyperplane as we like.

7.3. Theorem (Finite speed of regular fronts). Assume that $u_{I} \in C^{\alpha}$ and assume that all of the points of $\partial A\left(t_{0}\right) \cap B_{1}$ belong to $\mathcal{G}(\eta r, r)$ for some small $\eta<1 / 10$ and all $r<1$. Assume also that $0 \in \partial A\left(t_{0}\right)$ and that the set $\partial A\left(t_{0}\right) \cap B_{1}$ is given by the graph of a $C^{1, \alpha}$ function. Then there exist constants $\gamma>0$ and $\epsilon>0$ such that for any $t \in\left(t_{0}, t_{0}+\epsilon\right]$ we have

$$
\partial A(t) \cap B_{\gamma\left(t-t_{0}\right)} \neq \varnothing .
$$

Proof. Fix $t \in\left(t_{0}, t_{0}+\epsilon\right]$ (with $0<\epsilon \leq 1$ to be determined later), and as in the proof of Theorem 6.5 we let

$$
\Psi(x):=W(x, t)-W\left(x, t_{0}\right),
$$

and let $L$ be the set $A(t) \backslash A\left(t_{0}\right)$. Again as in Theorem 6.5 we have

$$
0 \leq W(x, t)-W\left(x, t_{0}\right) \leq\left(t-t_{0}\right)\|p\|_{L^{\infty}\left(\partial D \times\left[0, t_{0}+1\right]\right)} .
$$

Now we have a bound on the $C^{1, \alpha}$ norm of $\partial A\left(t_{0}\right) \cap B_{3 / 4}$ from obstacle problem regularity theory and our assumptions, but we need to choose $\epsilon>0$ sufficiently small to ensure that there is a bound on the $C^{1, \alpha}$ norm of $\partial A(t) \cap B_{5 / 8}$ for all $t \in\left(t_{0}, t_{0}+\epsilon\right]$. This estimate can also be obtained by standard regularity and stability theory for the obstacle problem. Next note that $W\left(x, t_{0}\right) \equiv 0$ in $L$, and it follows from regularity theory for the obstacle problem near smooth free boundary points that

$$
W(x, t) \sim \operatorname{dist}\left(x, A(t)^{c}\right)^{2}
$$

(see $\left[\mathrm{C}\right.$ or $[\mathrm{Bl}]$ ). So by our choice of $\epsilon$ we can assume that this result holds in $B_{5 / 8}$. 
Next define $v$ as the solution of the following boundary value problem:

$$
\begin{aligned}
\Delta v & =0 & & \text { in } A(t), \\
v & =0 & & \text { on } \partial A(t) \backslash \partial D, \\
v & =-\Psi & & \text { on } \partial D .
\end{aligned}
$$

Now if $\Theta:=v+\Psi$, then $\Delta \Theta \geq 0$ in $A(t)$, and $\Theta \equiv 0$ on $\partial A(t)$, so it follows from the weak maximum principle that $\Theta<0$ in $A(t)$. This leads to the inequalities:

$$
W(x, t)+v(x) \leq W\left(x, t_{0}\right) \leq W(x, t) .
$$

Now from our uniform $C^{1, \alpha}$ estimate for $\partial A(t) \cap B_{5 / 8}$ with $t \in\left(t_{0}, t_{0}+\epsilon\right]$ we can use the intermediate Schauder estimates of Gilbarg and Hörmander (see $\mathrm{GH}$ ) to conclude that in $B_{1 / 2}$ we have the estimate

$$
v(x) \sim\left(t-t_{0}\right)\|p\|_{L^{\infty}\left(\partial D \times\left[0, t_{0}+1\right]\right)} \operatorname{dist}\left(x, A(t)^{c}\right) .
$$

If we let $d(x)$ denote the distance from $x$ to $A(t)^{c}$, then we can use Equations (17.8), (7.7), and (7.5) to get:

$$
C d(x)\left[d(x)-\tilde{C}\left(t-t_{0}\right)\right] \leq W\left(x, t_{0}\right) \leq W(x, t)
$$

for a constant $\tilde{C}>0$ which depends only on the regularity estimates, and not on the particular solution otherwise. So if $d(x)>\tilde{C}\left(t-t_{0}\right)$, then it forces $W\left(x, t_{0}\right)$ to be positive. This proves the theorem with $\gamma=\tilde{C}$.

7.4. Theorem (Convergence to smooth free boundaries). If $\partial A(t) \cap \overline{B_{1 / 2}}$ belongs to $C^{1, \alpha}\left(\overline{B_{1 / 2}}\right)$, then there exists an $m \in \mathbb{N}$ and $a C>0$ which depend only on the regularity of $\partial A(t) \cap \overline{B_{1 / 2}}$ and on $\|p\|_{L^{\infty}(\partial D)}$ such that

$$
d_{\mathcal{H}}\left(\partial A(t) \cap \overline{B_{1 / 4}}, \partial A^{(m)}(t) \cap \overline{B_{1 / 4}}\right) \leq \frac{C}{m} .
$$

Proof. This result follows immediately from Equations (5.12), (5.13), (5.14), and Theorem 5.4 of Bl].

\section{ACKNOWLEDGMENTS}

The first author wishes to thank Kansas State University for their hospitality while the bulk of this paper was written. He also wishes to thank Ryan Berndt for a useful conversation. The second author is indebted to Juan Luis Vázquez for introducing her to the Hele-Shaw problem. She was partially supported by NSF EPSCoR Grant \#530517 under agreement NSF32169/KAN32170, and some of the first author's visits to Kansas State University were also supported by this grant. The second author received further support from NSF grant \#050391, and the first author received further support from NSF grant \#0501770. All three authors wish to thank Barbara Korten for her patience and support while this paper was written.

\section{REFERENCES}

[AK] D. Andreucci and M. Korten, Initial traces of solutions to a one-phase Stefan problem in an infinite strip, Rev. Mat. Iberoamericana, 9(1993), no. 2, 315-332. MR.1232846 (94m:35319)

[BF] B. Bazaliy and A. Friedman, Global existence and asymptotic stability for an ellipticparabolic free boundary problem: An application to a model of tumor growth, Indiana Univ. Math. J., 52(2003), no. 5, 1265-1304. MR2010327 (2004j:35302) 
[BBH] Ph. Bénilan, L. Boccardo, and M.A. Herrero, On the limit of solutions of $u_{t}=\Delta u^{m}$ as $m \rightarrow \infty$, Rend. Mat. Univ. Pol. Torino, Fascicolo Speciale (1989), Nonl. PDE's, 1-13. MR 1155452 (93c:35069)

[BEG] Ph. Bénilan, L.C. Evans, and R.F. Gariepy, On some singular limits of homogeneous semigroups, J. Evol. Eq., 3(2003), no. 2, 203-214. MR.1980973 (2004d:34122)

[Bl] I. Blank, Sharp results for the regularity and stability of the free boundary in the obstacle problem, Indiana Univ. Math. J. 50(2001), no. 3, 1077-1112. MR 1871348(2002i:35213)

[Bo] J.E. Bouillet, Nonuniqueness in $L^{\infty}$ : An example, Lecture notes in pure and applied mathematics, Vol. 148, Marcel Dekker, Inc., (1993). MR.1236685 (94h:35099)

[BoKM] J.E. Bouillet, M.K. Korten, and V. Márquez, Singular limits and the "Mesa" problem, Rev. Un. Mat. Argentina, 41(1998), no. 1, 27-40. MR1682193 (2000g:35236)

[C] L.A. Caffarelli, The obstacle problem revisited, J. Fourier Anal. Appl., 4(1998), no. 4-5, 383-402. MR1658612 (2000b:49004)

[CF] L.A. Caffarelli and A. Friedman, Asymptotic behavior of solutions of $u_{t}=\Delta u^{m}$ as $m \rightarrow \infty$, Indiana Univ. Math. J., 36(1987), no. 4, 203-224. MR916741(88m:35075)

[CJK] S. Choi, D. Jerison, and I. Kim, Regularity for the one-phase Hele-Shaw problem from a Lipschitz initial surface, Amer. J. Math. 129 (2007), 527-582. MR 2306045 (2008d:35238)

[DK] D. Danielli and M.K. Korten, On the pointwise jump condition at the free boundary in the 1-phase Stefan problem, Comm. Pure Appl. Anal., 4(2005), no. 2, 357-366. MR2149521 (2006c:35304)

[DL] P. Daskalopoulos and K. Lee, All time smooth solutions of the one-phase Stefan problem and the Hele-Shaw flow, Comm. PDE, 29(2004), no. 1-2, 71-88. MR2038144 (2004m:35283)

[DB] E. DiBenedetto, Continuity of weak solutions to certain singular parabolic equations, Ann. Mat. Pura Appl., (4), CXXX(1982), 131-176. MR663969 (83k:35045)

[DF] E. DiBenedetto and A. Friedman, The ill-posed Hele-Shaw model and the Stefan problem for supercooled water, Trans. Amer. Math. Soc., 282(1984), no. 1, 183-204. MR728709 (85g:35121)

[EHKO] C.M. Elliott, M.A. Herrero, J.R. King, and J.R. Ockendon, The mesa problem: Diffusion patterns for $u_{t}=\nabla \cdot\left(u^{m} \nabla u\right)$ as $m \rightarrow+\infty$, IMA J. Appl. Math., 37(1986), no. 2, 147154. MR983523 (89m:76061)

[EJ] C.M. Elliott and V. Janovský, A variational inequality approach to the Hele-Shaw flow with a moving boundary, Proc. Royal Soc. Edinburgh, Sect. 88A, 93-107. MR611303 (82d:76031)

[ES] J. Escher and G. Simonett, Classical solutions of multidimensional Hele-Shaw models, SIAM J. Math. Anal., 28(1997), 1028-1047. MR1466667 (98i:35213)

[FH] A. Friedman and S. Huang, Asymptotic behavior of solutions of $u_{t}=\Delta \phi_{m}(u)$ as $m \rightarrow \infty$ with inconsistent initial values, Analyse mathématique et applications, Gauthier-Villars, Paris, (1988), 165-180. MR956958 (89m:35027)

[GQV] O. Gil, F. Quirós, and J.L. Vázquez, Zero specific heat limit and large time asymptotics for the one-phase Stefan problem, preprint.

[GH] D. Gilbarg and L. Hörmander, Intermediate Schauder estimates, Arch. Ration. Mech. Anal., 74(1980), no. 4, 297-318. MR.588031 (82a:35038)

[GT] D. Gilbarg and N. Trudinger, Elliptic Partial Differential Equations of Second Order, Reprint of the 1998 Edition, Springer-Verlag, New York, 2001. MR1814364 (2001k:35004)

[I] N. Igbida, The mesa-limit of the porous-medium equation and the Hele-Shaw problem, Diff. Int. Eqns., 15(2002), no. 2, 129-146. MR1870466 (2002m:35119)

[JK] D. Jerison and I. Kim, The one-phase Hele-Shaw problem with singularities, J. Geom. Anal., 15(2005), no. 4, 641-668. MR2203166 (2006j:35247)

[Ki] I. Kim, Regularity of the free boundary for the one-phase Hele-Shaw problem, J. Diff. Eq., 223(2006), no. 1, 161-184. MR2210142(2006j:35248)

[KLV] J.R. King, A.A. Lacey, and J.L. Vázquez, Persistence of corners in Hele-Shaw flow, Euro. J. Appl. Math., 6(1995), no. 5, 455-490. MR1363758(97a:76037)

[K] M.K. Korten, Nonnegative solutions of $u_{t}=\Delta(u-1)_{+}:$Regularity and uniqueness for the Cauchy problem, Nonl. Anal., Th., Meth., and Appl., 27(1996), no. 5, 589-603. MR:1396031 (97h:35089) 
[KM] M.K. Korten and C.N. Moore, Regularity for solutions of the two-phase Stefan problem, Comm. Pure Appl. Anal. 7 (2008), 591-600. MR2379443

[MR] J.A. McGeough and H. Rasmussen, On the derivation of the quasi-steady model in electrochemical machining, J. Inst. Math. Appl., 13(1974), 13-21.

Department of Mathematics, Kansas State University, Manhattan, Kansas 66506

E-mail address: blanki@math.ksu.edu

Department of Mathematics, Kansas State University, Manhattan, Kansas 66506

E-mail address: marianne@math.ksu.edu

Department of Mathematics, Kansas State University, Manhattan, Kansas 66506

E-mail address: cnmoore@math.ksu.edu 